\title{
Exact and quasiexact solvability of second-order superintegrable quantum systems: I. Euclidean space preliminaries
}

\author{
E. G. Kalnins \\ Department of Mathematics, University of Waikato, Hamilton, New Zealand \\ W. Miller, Jr. \\ School of Mathematics, University of Minnesota, Minneapolis, Minnesota 55455 \\ G. S. Pogosyan \\ Laboratory of Theoretical Physics, Joint Institute for Nuclear Research, Dubna, \\ Moscow Region 141980, Russia \\ Departamento de Matématicas, CUCEI, Universidad de Guadalajara, Guadalajara, \\ Jalisco, Mexico
}

(Received 31 August 2005; accepted 20 January 2006; published online 8 March 2006)

\begin{abstract}
We show that second-order superintegrable systems in two-dimensional and threedimensional Euclidean space generate both exactly solvable (ES) and quasiexactly solvable (QES) problems in quantum mechanics via separation of variables, and demonstrate the increased insight into the structure of such problems provided by superintegrability. A principal advantage of our analysis using nondegenerate superintegrable systems is that they are multiseparable. Most past separation of variables treatments of QES problems via partial differential equations have only incorporated separability, not multiseparability. Also, we propose another definition of ES and QES. The quantum mechanical problem is called ES if the solution of Schrödinger equation can be expressed in terms of hypergeometric functions ${ }_{m} F_{n}$ and is QES if the Schrödinger equation admits polynomial solutions with coefficients necessarily satisfying a three-term or higher order of recurrence relations. In three dimensions we give an example of a system that is QES in one set of separable coordinates, but is not ES in any other separable coordinates. This example encompasses Ushveridze's tenth-order polynomial QES problem in one set of separable coordinates and also leads to a fourth-order polynomial QES problem in another separable coordinate set. (C) 2006 American Institute of Physics.
\end{abstract}

[DOI: $10.1063 / 1.2174237]$

\section{INTRODUCTION}

It is well known that $N$-dimensional nonrelativistic quantum systems described by the Hamiltonian

$$
\mathcal{H}=-\frac{1}{2} \sum_{i=1}^{N} \frac{\partial^{2}}{\partial x_{i}^{2}}+V\left(x_{1}, x_{2}, \ldots, x_{N}\right)
$$

are integrable if there exist $N$ linearly independent and global differential operators $\mathcal{I}_{\ell}, \ell$ $=0,1, \ldots, N-1$ and $\mathcal{I}_{0}=\mathcal{H}$, commuting with the Hamiltonian (1) and with each other

$$
\left[\mathcal{I}_{\ell}, \mathcal{H}\right]=0, \quad\left[\mathcal{I}_{\ell}, \mathcal{I}_{j}\right]=0, \quad \ell, j=1,2, \ldots, N-1 .
$$

This particular class of integrable systems is called (maximally) superintegrable (this term was introduced first by Rauch-Wojciechowski in Ref. 1) if it is integrable and, also, possesses $2 N-1$ 
functionally independent differential operators (integrals of motion). The additional $N-1$ integrals $\mathcal{L}_{k}$, commute with the Hamiltonian

$$
\left[\mathcal{L}_{k}, \mathcal{H}\right]=0, \quad k=1,2, \ldots, N-1,
$$

but not necessarily with each other. (These definitions have obvious classical analogs for the classical Hamiltonian.) Three examples of this kind have been well known for a long time, viz. the Kepler-Coulomb problem, the isotropic harmonic oscillator, and the nonisotropic oscillator with commensurable frequencies.

The existence of additional quantum integrals of motion for these systems leads to many interesting properties not shared by integrable systems. In classical mechanics the corresponding additional integrals of motion have the consequence that in the case of superintegrable systems in two dimensions and maximally superintegrable systems in three dimensions all finite trajectories are found to be periodic.

One of the most important properties for many superintegrable systems (particularly secondorder systems where there are $2 \mathrm{~N}-1$ functionally independent quadratic constants of the motion) is multiseparability, i.e., the separation of variables for the Hamilton-Jacobi and Schrödinger equations in more than one orthogonal coordinate system. ${ }^{2-8}$ (Each separable coordinate system is associated with $N$ commuting second-order constants of the motion.) For instance, the isotropic harmonic oscillator in three dimensions is separable in eight coordinate systems, namely in Cartesian, spherical, circular polar, circular elliptic, conical, oblate spheroidal, prolate spheroidal, and ellipsoidal coordinates. The Kepler-Coulomb potential is separable in four coordinate systems, namely in conical, spherical parabolic, and prolate spheroidal coordinates.

A systematic search for such systems in two- and three-dimensional Euclidean space was started in the pioneering work of Smorodinsky and Winternitz with collaborators in Refs. 9-11 and was continued in Ref. 12. Particularly, in Ref. 10 it was shown that in two-dimensional real Euclidean space there exist four superintegrable potentials, three of which could be considered as the singular generalization of Kepler-Coulomb, circular oscillator and anisotropic oscillator systems. These results were extended for two- and three-dimensional spaces with constant curvature (both positive and negative), ${ }^{13}$ and on the complex two-dimensional sphere and Euclidean space. ${ }^{14-18}$ The program is continuing for various conformally flat space spaces. ${ }^{19-30,5-7}$

In the last 15 years superintegrable systems have become a subject of investigation from many points of view: in Refs. 13, 18, 31, and 32 via the path integral approach, in Refs. 19, 21, and 22 by solving the Schrödinger equation with the help of the Niven ansatz, ${ }^{33}$ in Refs. $34-39$ from the purely algebraic approach, and generally in Ref. 40. As has been shown by a number of authors, many superintegrable systems generate an algebraic structure which may be considered as a nonlinear extension of the Lie algebra (in classical mechanics Poisson algebras), namely a quadratic algebra. The general form of quadratic algebras, which are encountered in the case of two-dimensional quantum superintegrable systems has been investigated. ${ }^{39,5,6}$

Particularly useful is the exact solvability of many superintegrable systems. Essentially, this means that after any separation of variables each of the separated ordinary differential equations admits an exact solution. However, the term exact solvability is defined differently by different authors. In Refs. 41 and 42 [see also the recent paper (Ref. 43)] we read that "an exactly solvable quantum mechanical system can be characterized by the fact that in its solution space one can indicate explicitly an infinite flag of functional linear spaces, which is preserved by the Hamiltonian" or the "Hamiltonian is exactly solvable if its spectrum can be calculated algebraically." Indeed, in spite of an "intuitive" understanding of the term exactly solvable, no universal definition exists up to now.

On the other hand, there are limiting cases of well-known one-dimensional exactly solvable systems, namely the harmonic oscillator and Coulomb problems with $\gamma / x^{2}(\gamma>-1 / 4)$ interaction, Morse potential, trigonometric and modified Pöschl-Teller potentials, trigonometric and hyperbolic Manning-Rosen potentials, ${ }^{44,45}$ and the Natanson potential. ${ }^{46}$ All these potentials have the general property that the Schrödinger spectral problem has an explicit formula for the whole energy spectrum including the continuous spectrum, and the eigenfunctions (up to the asymptotic 
ansatz or gauge transformations ${ }^{41,42}$ ) are of hypergeometric type ${ }_{1} F_{1},{ }_{2} F_{1}$. For the bound states we have solutions in terms of classical polynomials ${ }^{47}$ whereas for continuous states just infinite series. Moreover, hypergeometric functions describe both the continuous quantum systems as well as the finite systems and appear also as solutions of related difference equations, for instance, the finite one- and two-dimensional oscillator expressed in terms of discrete variables polynomials, Krawchuk, Meixner, and Hahn. ${ }^{48}$ The standard definitions of exact solvability do not include many of these systems.

Thus, we propose another definition of exact solvability: a quantum mechanical system is called exactly solvable if the solutions of the Schrödinger equation can be expressed in terms of hypergeometric functions ${ }_{m} F_{n}$. (Basically, we are requiring that the coefficients in power series expansions of the solutions satisfy two-term recurrence relations, rather than recurrence relations of higher order.) It is obvious that an $\mathrm{N}$-dimensional Schrödinger equation is exactly solvable if it is separable in some coordinate systems and each of the separated equations is exactly solvable. Further, we say that a superintegrable system is exactly solvable if it is exactly solvable in at least one system of coordinates.

At first sight, such a definition of exactly solvable problems may seem too narrow, but it leads us to distinguish two kinds of models: (1) those which it is possible to study analytically and (2) those which can be solved numerically via the solution of algebraic equations.

The process of separation of variables in the $N$-dimensional Schrödinger equation leads to ordinary differential equations having as solutions many of the special functions of mathematical physics. A complication of the separated equations involves the $N$ separation constants. In general we have a multiparameter eigenvalue problem. ${ }^{49}$ It is possible to distinguish three different cases, namely when there is complete, partial or nonseparability of the separation constants. It is obvious that in the case of complete separability (of separation constants) the initial $\mathrm{N}$-dimensional Schrödinger equation splits into $N$ independent second-order differential equations, each involving a single separation parameter. This situation occurs, for instance, in the case of separation of variables in the Helmholtz (free Schrödinger equation, which is also superintegrable) or the Schrödinger equation for the harmonic oscillator in Cartesian coordinates. The second "extremal" case, when complete nonseparability exists, is realized, in separation of variables for the same problems but in ellipsoidal coordinates. In the last case each separated second-order differential equation contains simultaneously all separation constants (usually depending on dimensional or nondimensional parameters), ${ }^{3,4}$ for which the simultaneous quantization becomes nontrivial.

The standard method of solution of a second-order ordinary differential equation, obtained after separation of variables in $N$-dimensional Schrödinger equations, involves (after taking into account the asymptotic ansatz) expansions around one of the singular points of the differential equation (the standard power series method, ${ }^{50}$ or the so-called Hill-determinant method ${ }^{51}$ ). The problem reduces to the solution of the recurrence relations for the expansion coefficients. If one can express the equation in a form such that the coefficients obey a two-term recurrence relation, then the corresponding solution can be written in closed or analytic form or in terms of hypergeometric functions and we have an exactly solvable problem. Such situations occur when separation of variables for superintegrable systems is possible in subgroup type coordinate (spherical, cylindrical, and Cartesian) ${ }^{52}$ and often in parabolic type coordinates. This method is also powerful when separation of variables is possible in nonsubgroup systems of coordinates such as spheroidal or elliptic types. In this case we arrive at high-order recurrence relations, the subsequent analysis of which allows us to investigate the behavior of the solution and to determine if polynomial solutions exist.

There is another general approach for solving the Schrödinger equation by exploring the Niven-type ansatz, ${ }^{33}$ based on the existence of polynomial solutions. According to this method the complete solution can be constructed without direct separation of variables and computed in terms of the zeros of the corresponding polynomial. This method has been used in Refs. 19, 21, and 22 for the investigation of two- and three-dimensional superintegrable systems in Euclidean and curved spaces. We illustrate the difference between systems that are merely separable and those 
that are superintegrable. Consider the problem of motion in the plane for a charged particle with two fixed Coulomb centers with coordinates $( \pm D / 2,0)$ (the so-called plane two center problem)

$$
V(x, y)=-\frac{\alpha_{1}}{\sqrt{y^{2}+(x+D / 2)^{2}}}-\frac{\alpha_{2}}{\sqrt{y^{2}+(x-D / 2)^{2}}} .
$$

This system is not superintegrable and separation of variables is possible only in two-dimensional elliptic coordinates [see Eq. (70)]. Upon the substitution $\psi\left(\nu, \mu ; D^{2}\right)=X\left(\nu ; D^{2}\right) Y\left(\mu ; D^{2}\right)$ and the separation constant $A(D)$, the Schrödinger equation splits into a system of two ordinary differential equations

$$
\begin{gathered}
\frac{d^{2} X}{\mathrm{~d} \nu^{2}}+\left[\frac{D^{2} E}{2} \cosh ^{2} \nu+D\left(\alpha_{1}+\alpha_{2}\right) \cosh \nu+A(D)\right] X=0, \\
\frac{d^{2} Y}{\mathrm{~d} \mu^{2}}-\left[\frac{D^{2} E}{2} \cos ^{2} \mu+D\left(\alpha_{1}-\alpha_{2}\right) \cos \mu+A(D)\right] Y=0 .
\end{gathered}
$$

Both Eqs. (5) and (6) belong to the class of nonexactly solvable problems. In general polynomial solutions do not exist even for the case of discrete spectrum $E<0$ (to be completely correct let us note that polynomial solutions exist only for special values of parameters $\alpha_{1}, \alpha_{2}$, and $R$ ), and each of the wave functions $X\left(\nu ; D^{2}\right)$ and $Y\left(\mu ; D^{2}\right)$ is expressed as an infinite series with a three-term recurrence relation.

Let us now set $\alpha_{2}=0$. Then the potential (4) transforms to the ordinary two-dimensional (2D) hydrogen atom problem, which is well known as a superintegrable system ${ }^{53-55}$ with dynamical symmetry group $\mathrm{SO}(3)$, and admits separation of variables in three systems of coordinates: polar, parabolic, and elliptic. In this case we can see that the separation equations (5) and (6), namely

$$
\begin{gathered}
\frac{d^{2} X}{\mathrm{~d} \nu^{2}}+\left[\frac{D^{2} E}{2} \cosh ^{2} \nu+D \alpha_{1} \cosh \nu+A(D)\right] X=0, \\
\frac{d^{2} Y}{\mathrm{~d} \mu^{2}}-\left[\frac{D^{2} E}{2} \cos ^{2} \mu+D \alpha_{1} \cos \mu+A(D)\right] Y=0
\end{gathered}
$$

transform into each other by the change $\mu \leftrightarrow i \nu$. Thus separation of variables in elliptic coordinates for the 2D hydrogen atom gives two functionally identical one-dimensional Schrödinger type equations with two parameters: coupling constant $E$ and energy $A(D)$ (correspondingly energy and separation constant for 2D), but one defined on the real and the other on the imaginary axis. In other words, instead of the systems of differential equations (7) and (8), the problem reduces to solving only one of the equations (7) or (8) for which the "domain of definition" is the complex plane. The requirement of finiteness for the wave functions in the complex plane permits only polynomial solutions (see for details Ref. 56). As a result we obtain simultaneous quantization of the energy spectrum

$$
E_{n}=-\frac{\alpha_{1}^{2}}{2(n+1 / 2)^{2}}, \quad n=0,1,2, \ldots
$$

and the elliptic separation constant $A_{s}(D)$ where $s=0,1,2, \ldots, n$ (as a solution of an $n$ th-degree algebraic equation). The polynomial solution is defined by a finite series with three-term recurrence relations for the coefficients. They cannot be considered as exactly solvable and can be investigated only numerically. A similar situation occurs, for instance, in the case of the two-center problem in three-dimensional Euclidean space (the so-called prolate spheroidal radial and angular Coulomb wave functions) ${ }^{57}$ and three-dimensional sphere (Heun wave functions), ${ }^{58}$ where after eliminating one of the Coulomb centers the problems reduce to superintegrable systems admitting only polynomial solutions. These (and many other) examples suggest a deep connection of the 
notion of superintegrability and existence of polynomial solutions of the corresponding Schrödinger equation.

We note that each of equations (7) or (8) has the form of a one-dimensional Schrödinger equation with the parameter $E$ and eigenvalue $A(D)$, and could be separately considered in the regions $\mu \in[0,2 \pi]$ or $\nu \in[0, \infty)$, correspondingly. Then for arbitrary values of constant $E$ [for example when $E_{n}=0(n \rightarrow \infty)$ the equations (7) and (8) transform to periodic and modified Mathieu equations, which are nonexactly solvable] the solutions of Eqs. (7) or (8) expressed via infinite series and only on the "energy surface" of the 2D hydrogen atom (9), split into polynomial and nonpolynomial sectors (each of these sectors is noncomplete) and for fixed number $n$, only some of the eigenvalues $A_{s}(D)(s=0,1,2, \ldots, n)$ can be calculated from an $n$ th-degree algebraic equation. We can say that Eqs. (7) and (8) "remember" their polynomial solutions. It is obvious that the spectrum of $A_{s}(D),(s=0,1,2 \ldots, n)$ and occurrence of polynomial solutions of each of the equations (7) and (8) coincides with the eigenvalues of separation constants and the wave function after the reduction to one of the regions $\mu \in[0,2 \pi]$ or $\nu \in[0, \infty)]$ for the $2 \mathrm{D}$ hydrogen atom.

These phenomena have been intensively discussed in the literature in the late 1980s and called quasiexact solvability (this term was first introduced by Turbiner and Ushveridze in Ref. 59) and models of this type called quasiexactly solvable systems ${ }^{60-62}$ (see also Ref. 63 and references therein). The crucial example that stimulated the investigation of quasiexactly solvable systems is the Hamiltonian (1) with anharmonic potential

$$
V(x)=\frac{1}{2} \omega^{2} x^{6}+2 \beta \omega^{2} x^{4}+\left(2 \beta^{2} \omega^{2}-2 \delta \omega-\lambda\right) x^{2}+2 \frac{\left(\delta-\frac{1}{4}\right)\left(\delta-\frac{3}{4}\right)}{x^{2}},
$$

where $\omega, \beta, \delta>1 / 2$ and $\lambda$ are constants. As noticed by many authors, ${ }^{64-66}$ this system admits polynomial solutions only for special values of constant $\lambda=\omega(2 n+1)(n=0,1,2 \ldots)$

$$
\Psi(x) \approx x^{2 \delta-1 / 2} e^{-(\omega / 4) x^{4}-\beta \omega x^{2}} P_{n}\left(x^{2}\right) .
$$

There are different approaches to the investigation of quasiexactly solvable systems. In the algebraic approach formulated by Turbiner in Ref. 60 quasiexact solvability is explained in terms of a "hidden symmetry algebra" $\mathrm{sl}(2, R)$. [This is not a hidden dynamical symmetry in the usual sense because the Hamiltonian (12) belongs to the enveloping algebra but is not a Casimir operator.] More precisely this means the following: The one-dimensional Hamiltonian (1) after suitable changes of variable $z=\xi(x)$ and "gauge transformation" $H=e^{-\alpha(z)} \mathcal{H} e^{\alpha(z)}$ can be written in the form

$$
H=\sum_{a, b=0, \pm} C_{a b} J_{a} J_{b}+\sum_{a=0, \pm} C_{a} J_{a}
$$

where the first-order differential operators $\left\{J_{ \pm}, J_{0}\right\}$ satisfy the commutation relations for $\operatorname{sl}(2, R) .^{60}$

The above mentioned analysis for the $2 \mathrm{D}$ hydrogen atom shows that, despite the elegance of the algebraic approach, the phenomena of quasiexactly solvability has deeper roots than can be explained via the "one-dimensional" model (12). Other examples are the hydrogen atom and oscillator problems on two- and three-dimensional spheres ${ }^{19,67}$ and two-dimensional hyperboloids, ${ }^{22}$ which generate not only hyperbolic and trigonometric but elliptic quasiexactly solvable systems (see also Refs. 68, 56, 69, and 70). We should also mention Lamé polynomials. They come from separation of variables for the Helmholtz (also superintegrable!) or Schrödinger equation in elliptic coordinates on the two-dimensional sphere. As also determined in Ref. 37 (without showing the mechanism of this phenomena) some of the quasiexactly solvable systems can be obtained through dimensional reduction from two- and three-dimensional superintegrable models with quadratic invariants (second-order superintegrability).

A second approach, known as analytic, was formulated by Ushveridze (see, for example, Refs. 61-63) and represents a one-dimensional reduction of the Niven-Stieltjes method for solving multiparameter spectral problems such as the generalized Lamé equation (or ellipsoidal equation). ${ }^{33}$ The solution in this method is determined by the zeros of polynomials $P_{n}\left(x^{2}\right)$. Then the wave function (11) can be rewritten in the form 


$$
\Psi(x) \approx x^{2 \delta-1 / 2} e^{-(\omega / 4) x^{4}-\beta \omega x^{2}} \prod_{i=0}^{n}\left(x^{2}-\xi_{i}\right),
$$

where the numbers $\left(\xi_{1}, \xi_{2}, \ldots, \xi_{n}\right)$ satisfy a system of $n$ algebraic equations (see Sec. II C). According to the oscillation theorem, the number of zeros in the physical interval $\xi_{i} \in[0, \infty)$ enumerates the ground state and first $n$ excitations, described in terms of all zeros (complete solutions of the systems of algebraic equations and including nonphysical section $\left.\xi_{i} \in(-\infty, 0]\right)$ as

$$
E=4 \delta\left[\beta \omega+\sum_{i=1}^{n} \frac{1}{\xi_{i}}\right]
$$

Two natural questions occur in this approach: what is the physical meaning of the negative zeros $\xi_{i}$, and why in the correct formula for the energy spectrum (14) do $n$ zeros of the polynomial $P_{n}\left(x^{2}\right)$ appear?

With this paper we begin an investigation of second-order superintegrable systems on constant curvature spaces (Euclidean, sphere, hyperboloid and pseudo-Euclidean) based on the superintegrability and direct solutions of the Schrödinger equation. We pay special attention to nonsubgroup type coordinates and prove the existence of polynomial solutions for several of these systems. We demonstrate that quasiexact solvability is directly related with multiseparability of second-order superintegrable systems, on one hand, and with the presence of polynomial solutions for these systems on the other.

The first part of this paper is devoted to two (singular anisotropic and singular circular oscillators) from the four possible superintegrable systems in two-dimensional real Euclidean space (see, for example, Ref. 19). The other two systems may be transformed (only for the discrete spectrum) to the singular circular oscillator (for $V_{3}$ ) or ordinary shifted oscillator (for $V_{4}$ ) systems by the help of the Levi-Civita mapping, ${ }^{71}$ so are less fundamental for our purposes. In the second part of the paper we give some examples of superintegrable systems in three dimensions that reinforce our definitions of exact and quasiexact solvability. In particular we exhibit a quasiexactly solvable superintegrable system which is not at the same time exactly solvable in any separable set of coordinates. In one set of separable coordinates this provides deeper insight into an example of Ushveridze, ${ }^{63}$ p. 155 (the tenth-order polynomial QES problem) and also leads to a fourth-order polynomial QES problem in another separable coordinate set. In addition we indicate precisely how the eigenvalues of the symmetry operators which describe separation can be calculated from a determinant condition. For these examples we will work with complex superintegrable systems and not address the relatively simple issue of determining the distinct real restrictions of the complex spaces. These examples greatly clarify the concepts and show how the extension to $N$ dimensions can be achieved.

\section{THE SINGULAR ANISOTROPIC OSCILLATOR}

Let us first consider the potential $\left(k_{2}>0\right)$

$$
V_{1}(x, y)=\frac{1}{2} \omega^{2}\left(4 x^{2}+y^{2}\right)+k_{1} x+\frac{k_{2}^{2}-\frac{1}{4}}{2 y^{2}}
$$

the singular anisotropic oscillator. The Schrödinger equation has the form

$$
\left(\frac{\partial^{2}}{\partial x^{2}}+\frac{\partial^{2}}{\partial y^{2}}\right) \Psi+\left[2 E-\omega^{2}\left(4 x^{2}+y^{2}\right)-2 k_{1} x-\frac{k_{2}^{2}-\frac{1}{4}}{y^{2}}\right] \Psi=0 .
$$

For $k_{2}>1 / 2$ the singular term at $y=0$ is repulsive and the motion takes place only on one of the half planes $(-\infty<x<\infty, y>0)$ or $(-\infty<x<\infty, y<0)$, whereas for $0<k_{2}<1 / 2$ in whole plane $(x, y)$. The Schrödinger equation separates in two systems: Cartesian and parabolic coordinates. 


\section{A. Cartesian bases}

Separation of variables for Eq. (16) in Cartesian coordinates leads to the two independent one-dimensional Schrödinger equations

$$
\begin{gathered}
\frac{d^{2} \psi_{1}}{\mathrm{~d} x^{2}}+\left(2 \lambda_{1}-4 \omega^{2} x^{2}-2 k_{1} x\right) \psi_{1}=0, \\
\frac{d^{2} \psi_{2}}{\mathrm{~d} y^{2}}+\left(2 \lambda_{2}-\omega^{2} y^{2}-\frac{k_{2}^{2}-\frac{1}{4}}{y^{2}}\right) \psi_{2}=0,
\end{gathered}
$$

where

$$
\Psi\left(x, y ; k_{1}, \pm k_{2}\right)=\psi_{1}\left(x ; k_{1}\right) \psi_{2}\left(y ; \pm k_{2}\right)
$$

and $\lambda_{1}, \lambda_{2}$ are Cartesian separation constants with $\lambda_{1}+\lambda_{2}=E$.

Equation (18) represents the well-known linear singular oscillator system (see for instance Refs. 72 and 73 and Refs. 10, 20, and 74). It is an exactly solvable problem and has been used in many applications, for example, as a model in $N$-body problems, ${ }^{75}$ or fractional statistics and anyons. ${ }^{76,77}$ The complete set of orthonormalized eigenfunctions, (on 1/2) in the interval $0<y$ $<\infty$ of Eq. (18), can be expressed in terms of finite confluent hypergeometric series or Laguerre polynomials

$$
\psi_{n_{2}}\left(y ; \pm k_{2}\right)=\sqrt{\frac{2 \omega^{\left(1 \pm k_{2}\right)} n_{2} !}{\Gamma\left(n_{2} \pm k_{2}+1\right)}} y^{1 / 2 \pm k_{2}} e^{-1 / 2 \omega y^{2}} L_{n_{2}}^{ \pm k_{2}}\left(\omega y^{2}\right),
$$

where $\lambda_{2}=\omega\left(2 n_{2}+1 \pm k_{2}\right)$. We assume that the positive sign at the $k_{2}$ must be taken if $k_{2}>\frac{1}{2}$ and both the positive and the negative sign must be taken if $0<k_{2}<\frac{1}{2}$, so that the polynomials have finite norm. Let us also note that unlike the potential (15) the wave function is not invariant under the replacement $k_{2} \rightarrow-k_{2}$ and splits into two families of solutions that transform to one another under this change.

The second equation (17) easily transforms to the ordinary one-dimensional oscillator problem. In terms of Hermite polynomials the orthonormal solutions (in region $-\infty<x<\infty$ ) are

$$
\psi_{n_{1}}\left(x ; k_{1}\right)=\left(\frac{2 \omega}{\pi}\right)^{1 / 4} \frac{e^{-\omega z^{2}}}{\sqrt{2^{n_{1}} n_{1} !}} H_{n_{1}}(\sqrt{2 \omega z}), \quad z=x+\frac{k_{1}}{4 \omega^{2}},
$$

where $\lambda_{1}=\omega\left(2 n_{1}+1\right)-\left(k_{1}^{2} / 8 \omega^{2}\right)$. Thus the complete energy spectrum is

$$
E=\lambda_{1}+\lambda_{2}=\omega\left[2 n+2 \pm k_{2}\right]-\frac{k_{1}^{2}}{8 \omega^{2}}, \quad n=n_{1}+n_{2}=0,1,2, \ldots
$$

and the degree of degeneracy for fixed principal quantum number $n$ is $(n+1)$. Finally note that the separation of variables in Cartesian coordinates leads to two exactly solvable one-dimensional Schrödinger equations and the complete wave function may be constructed with the help of formulas (20), (21), and (19).

\section{B. Parabolic bases}

\section{Separation of variables}

Parabolic coordinates $\xi$ and $\eta$ are connected with the Cartesian $x$ and $y$ by

$$
x=\frac{1}{2}\left(\xi^{2}-\eta^{2}\right), \quad y=\xi \eta, \quad \xi \in \mathbf{R}, \quad \eta>0 .
$$

The Laplacian and the two-dimensional volume element are given by 


$$
\Delta=\frac{\partial^{2}}{\partial x^{2}}+\frac{\partial^{2}}{\partial y^{2}}=\frac{1}{\xi^{2}+\eta^{2}}\left(\frac{\partial^{2}}{\partial \xi^{2}}+\frac{\partial^{2}}{\partial \eta^{2}}\right), \quad \mathrm{d} v=\mathrm{d} x \mathrm{~d} y=\left(\xi^{2}+\eta^{2}\right) \mathrm{d} \xi \mathrm{d} \eta .
$$

The Schrödinger equation in parabolic coordinates (23) is

$$
\frac{1}{\xi^{2}+\eta^{2}}\left(\frac{\partial^{2} \Psi}{\partial \xi^{2}}+\frac{\partial^{2} \Psi}{\partial \eta^{2}}\right)+\left[2 E-\omega^{2}\left(\xi^{4}-\xi^{2} \eta^{2}+\eta^{4}\right)-k_{1}\left(\xi^{2}-\eta^{2}\right)-\frac{k_{2}^{2}-\frac{1}{4}}{\xi^{2} \eta^{2}}\right] \Psi=0 .
$$

Upon substituting

$$
\Psi(\xi, \eta)=X(\xi) Y(\eta)
$$

and introducing the parabolic separation constant $\lambda$, the equation (25) splits into two ordinary differential equations,

$$
\begin{gathered}
\frac{d^{2} X}{\mathrm{~d} \xi^{2}}+\left(2 E \xi^{2}-\omega^{2} \xi^{6}-k_{1} \xi^{4}-\frac{k_{2}^{2}-\frac{1}{4}}{\xi^{2}}\right) X=-\lambda X, \\
\frac{d^{2} Y}{\mathrm{~d} \eta^{2}}+\left(2 E \eta^{2}-\omega^{2} \eta^{6}+k_{1} \eta^{4}-\frac{k_{2}^{2}-\frac{1}{4}}{\eta^{2}}\right) Y=+\lambda Y .
\end{gathered}
$$

Equations (26) and (27) are transformed into one another by change $\xi \leftrightarrow i \eta$. We have

$$
\Psi(\xi, \eta ; E, \lambda)=C(E, \lambda) Z(\xi ; E, \lambda) Z(i \eta ; E, \lambda),
$$

where $C(E, \lambda)$ is the normalization constant determined by the condition

$$
\int_{0}^{\infty} \mathrm{d} \eta \int_{-\infty}^{\infty} \mathrm{d} \xi\left(\xi^{2}+\eta^{2}\right)|\Psi(\xi, \eta ; E, \lambda)|^{2}=1
$$

and the function $Z(\mu ; E, \lambda)$ is a solution of the equation

$$
\left[-\frac{d^{2}}{\mathrm{~d} \mu^{2}}+\left(\omega^{2} \mu^{6}+k_{1} \mu^{4}-2 E \mu^{2}+\frac{k_{2}^{2}-\frac{1}{4}}{\mu^{2}}\right)\right] Z(\mu ; E, \lambda)=\lambda Z(\mu ; E, \lambda) .
$$

Thus, at $\mu \in(-\infty, \infty)$ we have Eq. (26) and at $\mu \in[0, i \infty)$ —Eq. (27). Note that in the complex $\mu$ domain the "physical" region is just the two lines $\operatorname{Im} \mu=0$ and $\operatorname{Re} \mu=0, \operatorname{Im} \mu>0$. Our task is to find the solutions of Eq. (30) that are regular and decreasing as $\mu \rightarrow \pm \infty$ and $\mu \rightarrow i \infty$.

\section{Recurrence relations}

Consider now the equation (30). To solve it we make the substitution

$$
Z(\mu ; E, \lambda)=\exp \left(-\frac{\omega}{4} \mu^{4}-\frac{k_{1}}{4 \omega} \mu^{2}\right) \mu^{\frac{1}{2} \pm k_{2}} \psi(\mu ; E, \lambda),
$$

and obtain the differential equation

$$
\frac{d^{2} \psi}{\mathrm{d} \mu^{2}}+\left[\frac{2\left(\frac{1}{2} \pm k_{2}\right)}{\mu}-2 \omega \mu\left(\mu^{2}+\frac{k_{1}}{2 \omega^{2}}\right)\right] \frac{\mathrm{d} \psi}{\mathrm{d} \mu}+\left[2 \widetilde{E} \mu^{2}+\tilde{\lambda}\right] \psi=0,
$$

where

$$
\tilde{E}=E+\frac{k_{1}^{2}}{8 \omega^{2}}-\omega\left(2 \pm k_{2}\right), \quad \tilde{\lambda}=\lambda-\frac{k_{1}}{\omega}\left(1 \pm k_{2}\right) .
$$

Passing to a new variable $z=\mu^{2}$ in Eq. (32), we have 


$$
z \frac{d^{2} \psi}{\mathrm{d} z^{2}}+\left[\left(1 \pm k_{2}\right)-\omega z\left(z+\frac{k_{1}}{2 \omega^{2}}\right)\right] \frac{\mathrm{d} \psi}{\mathrm{d} z}+\left[\frac{1}{2} \widetilde{E} z+\frac{1}{4} \widetilde{\lambda}\right] \psi=0 .
$$

We express the wave function $\psi(z)$ in the form

$$
\psi(z ; E, \lambda)=\sum_{s=0}^{\infty} A_{s}(E, \lambda) z^{s} .
$$

The substitution (35) in Eq. (34) leads to the following three-term recurrence relation for the expansion coefficients $A_{s} \equiv A_{s}(E, \lambda)$,

$$
(s+1)\left(s+1 \pm k_{2}\right) A_{s+1}+\frac{1}{4}\left[\lambda-\frac{k_{1}}{\omega}\left(2 s+1 \pm k_{2}\right)\right] A_{s}+\frac{1}{2}\left[E+\frac{k_{1}}{8 \omega^{2}}-\omega\left(2 s \pm k_{2}\right)\right] A_{s-1}=0,
$$

with the initial conditions $A_{-1}=0$ and $A_{0}=1$.

As shown in the Appendix, the asymptotic behavior of the expansion coefficients $A_{s}$ $\equiv A_{s}(E, \lambda)$ for large $s$ is $A_{s} \sim \sqrt{\xi_{ \pm}} \sqrt{\omega^{s} / s !}$, depending on whether $s$ is even or odd, and

$$
\psi(z) \sim \sum \frac{\sqrt{\xi_{ \pm}}(\sqrt{\omega z})^{s}}{\sqrt{s !}} .
$$

Then we have for $z>0$ [the case of Eq. (26)],

$$
\sum \frac{\sqrt{\xi_{ \pm}}(\sqrt{\omega} z)^{s}}{\sqrt{s !}}>\sqrt{\sum \frac{\xi_{ \pm}\left(\omega z^{2}\right)^{s}}{s !}}=\xi_{ \pm} \cosh \left(\frac{\omega}{2} z^{2}\right)+\xi_{\mp} \sinh \left(\frac{\omega}{2} z^{2}\right) .
$$

This function does not belong to the Hilbert space. If $k_{1}>0$ then we must make the replacements $b_{s} \rightarrow-b_{s}$ and $\xi_{s} \rightarrow-\xi_{s}$. This has the effect of replacing $z$ by $-z$ in (37). Now the asymptotic solution is oscillatory. However, for $z<0$ [the case of Eq. (27)] the solution does not belong to the Hilbert space. The solution we have found is the minimal solution of the three-term recurrence relations. There is a linearly independent solution, but the coefficients grow more rapidly than the minimal solution coefficients.

\section{Energy spectrum and separation constant}

The function $Z(\mu)$ cannot converge simultaneously at large $\mu$ for real and imaginary $\mu$ and therefore the series (35) should be truncated in order to obtain convergence. The condition for series (35) to be truncated results in the energy spectrum (22) where now the coefficients $A_{s}$ $\equiv A_{s}^{n q}\left(k_{1}, \pm k_{2}\right)$ satisfy the relation

$$
(s+1)\left(s+1 \pm k_{2}\right) A_{s+1}+\beta_{s} A_{s}+\omega(n+1-s) A_{s-1}=0, \quad \beta_{s}=\frac{\lambda}{4}-\frac{k_{1}}{4 \omega}\left(2 s+1 \pm k_{2}\right) .
$$

The three-term recurrence relations (39) represent a homogeneous system of $n+1$ algebraic equations for $n+1$ coefficients $\left\{A_{0}, A_{1}, A_{2}, \ldots, A_{n}\right\}$. The requirement for the existence of a nontrivial solution leads to a vanishing of the determinant

$$
D_{n}(\lambda)=\left|\begin{array}{cccccc}
\beta_{0} & 1 \pm k_{2} & & & & \\
\omega n & \beta_{1} & 2\left(2 \pm k_{2}\right) & & \\
& & & 2 \omega & \beta_{n-1} & n\left(n \pm k_{2}\right) \\
& & & & \omega & \beta_{n}
\end{array}\right|=0 .
$$

The roots of the corresponding algebraic equation give us the $(n+1)$ eigenvalues of the parabolic separation constant $\lambda_{n}\left(k_{1}, \pm k_{2}\right)$. It is known that all roots for such determinants are real and 
distinct. ${ }^{78}$ Thus all values of the separation constant are real and can be enumerated with the help of the integer $q$, namely the values are $\lambda_{n}\left(k_{1}, \pm k_{2}\right) \rightarrow \lambda_{n q}\left(k_{1}, \pm k_{2}\right)$, where $0 \leqslant q \leqslant n$. The degeneracy for the $n$-energy state, as in the Cartesian case, equals $n+1$.

Note that Eq. (40) is invariant under the simultaneous transformation $k_{1} \rightarrow-k_{1}$ and $\lambda \rightarrow-\lambda$. Thus if one of the $\lambda=\lambda_{n}\left(k_{1}, \pm k_{2}\right)$ is a root of Eq. (40), then $\lambda=-\lambda_{n}\left(-k_{1}, \pm k_{2}\right)$ is also a root of the same equation. We see that for the odd energy state ( $n$-odd) the range of $\lambda_{n q}\left(k_{1}, \pm k_{2}\right)$ splits into two subsets $\lambda_{n q}^{(1)}$ and $\lambda_{n q}^{(2)}$ connected by the relation $\lambda_{n q}^{(1)}\left(k_{1}, \pm k_{2}\right) \leftrightarrow-\lambda_{n q^{\prime}}^{(2)}\left(-k_{1}, \pm k_{2}\right)$. For $n$-even, there exists the additional root $\lambda_{n q}\left(k_{1}, \pm k_{2}\right)=-\lambda_{n q}\left(-k_{1}, \pm k_{2}\right)$, which equals zero when $k_{1}=0$.

\section{Wave functions}

We will term the polynomial solutions of Eq. (34), or Eq. (32), as $M k_{n q}\left(z ; k_{1}, \pm k_{2}\right)$, and the function (31) as $T a_{n q}\left(z ; k_{1}, \pm k_{2}\right)$. [The notation $T a$ is in memory of Professor V. Ter-Antonyan (1942-2003).] Then the physical admissible solutions of Eq. (34) have the form

$$
M k_{n q}\left(z ; k_{1}, \pm k_{2}\right) \equiv \psi(z ; E, \lambda)=\sum_{s=0}^{n} A_{s}^{n q}\left(k_{1}, \pm k_{2}\right) z^{s},
$$

and the corresponding solution of Eq. (31) is

$$
T a_{n q}\left(\mu ; k_{1}, \pm k_{2}\right)=\exp \left(-\frac{\omega}{4} \mu^{4}-\frac{k_{1}}{4 \omega} \mu^{2}\right) \mu^{\frac{1}{2} \pm k_{2}} M k_{n q}\left(\mu^{2} ; k_{1}, \pm k_{2}\right) .
$$

Observe that parabolic wave functions (and also Cartesian wave functions) split into two classes and transform to each other via $k_{2} \rightarrow-k_{2}$. In the case $k_{2}=0$ (when the centrifugal term disappears), the solution (42) becomes an even and odd parity wave function under the exchange $\mu \rightarrow-\mu$.

It is known that there exists a direct connection between the quantum numbers $q$ and numbers of zeros of the polynomial (41) and, therefore, the eigenvalues of the separation constant $\lambda_{n q}\left(k_{1}, \pm k_{2}\right)$ may be ordered by the numbers of nodes of the wave function $T a_{n q}\left(\mu ; k_{1}, \pm k_{2}\right)$. Indeed we will see that these are orthogonal polynomials, hence, ${ }^{50}$ all the $n$ zeros of the $M k_{n q}\left(z ; k_{1}, \pm k_{2}\right)$ are situated on the real axis $-\infty<z<\infty$, and all zeros have multiplicity one. Assume that the separation constants $\lambda_{n q}\left(k_{1}, \pm k_{2}\right)$ are enumerated in ascending order, i.e.,

$$
\lambda_{n 0}\left(k_{1}, \pm k_{2}\right)<\lambda_{n 1}\left(k_{1}, \pm k_{2}\right)<\cdots<\lambda_{n, n-1}\left(k_{1}, \pm k_{2}\right)<\lambda_{n, n}\left(k_{1}, \pm k_{2}\right) .
$$

Then according to the oscillation theorem, ${ }^{79}$ the quantum number $q$ also enumerates the zeros of polynomials $M k_{n q}\left(z ; k_{1}, \pm k_{2}\right)$ in the region $z>0$, or the real axis of $\mu$. Let us now introduce two quantum numbers $q_{1}$ and $q_{2}$, which determine the zeros of polynomials $M k_{n q}\left(z ; k_{1}, \pm k_{2}\right)$ for $z$ $>0$ and $z<0$, correspondingly. Then $q_{1}+q_{2}=n$, and

$$
\lambda_{n q_{1}}\left(k_{1}, \pm k_{2}\right)=-\lambda_{n q_{2}}\left(-k_{1}, \pm k_{2}\right) .
$$

For $\mu=\xi$ the function (42) gives the solution of Eq. (26), and for $\mu=i \eta$ the solution of Eq. (27). Thus the parabolic wave function (28) can be written in the following way:

$$
\Psi_{n q_{1} q_{2}}\left(\xi, \eta ; k_{1}, \pm k_{2}\right)=C_{n q_{1} q_{2}}\left(k_{1}, \pm k_{2}\right) T a_{n q_{1}}\left(\xi ; k_{1}, \pm k_{2}\right) T a_{n q_{2}}\left(i \eta ; k_{1}, \pm k_{2}\right)
$$

\section{Orthogonality relations and normalization constant}

The wave functions (45) as eigenfunctions of Hamiltonians are orthogonal for quantum number $n$, or for $n \neq n^{\prime}$, 


$$
\int_{0}^{\infty} \mathrm{d} \eta \int_{-\infty}^{\infty} \mathrm{d} \xi\left(\xi^{2}+\eta^{2}\right) \Psi_{n^{\prime} q_{1} q_{2}}^{*}\left(\xi, \eta ; k_{1}, \pm k_{2}\right) \Psi_{n q_{1} q_{2}}^{*}\left(\xi, \eta ; k_{1}, \pm k_{2}\right)=0
$$

Because the energy spectrum is degenerate there exist additional orthogonality relations for quantum number $q$. Using the equations (26) and (27) it is easy to prove that for $q_{1} \neq q_{1}^{\prime}$ and $q_{2} \neq q_{2}^{\prime}$,

$$
\int_{-\infty}^{\infty} \mathrm{d} \xi T a_{n q_{1}^{\prime}}^{*}\left(\xi ; k_{1}, \pm k_{2}\right) T a_{n q_{1}}\left(\xi ; k_{1}, \pm k_{2}\right)=\int_{0}^{\infty} \mathrm{d} \eta T a_{n q_{2}^{\prime}}^{*}\left(i \eta ; k_{1}, \pm k_{2}\right) T a_{n q_{2}}\left(i \eta ; k_{1}, \pm k_{2}\right)=0 .
$$

Thus we have for $q \neq q^{\prime}$,

$$
\int_{0}^{\infty} \mathrm{d} \eta \int_{-\infty}^{\infty} \mathrm{d} \xi\left(\xi^{2}+\eta^{2}\right) \Psi_{n q_{1}^{\prime} \prime_{2}^{\prime}}^{*}\left(\xi, \eta ; k_{1}, \pm k_{2}\right) \Psi_{n q_{1} q_{2}}\left(\xi, \eta ; k_{1}, \pm k_{2}\right)=0
$$

Let us now calculate the normalization constant $C_{n q_{1} q_{2}}\left(k_{1}, \pm k_{2}\right)$. From the explicit form of the wave function $\Psi_{n q_{1} q_{2}}^{*}\left(\xi, \eta ; k_{1}, \pm k_{2}\right)$ and the normalization condition (29), it follows that

$$
\begin{aligned}
& \frac{1}{8}\left|C_{n q_{1} q_{2}}\left(k_{1}, \pm k_{2}\right)\right|^{2} \sum_{s, s^{\prime}, t, t^{\prime}=0}^{n}(-1)^{t+t^{\prime}} A_{s}^{n}\left(k_{1}, \pm k_{2}\right) A_{s^{\prime}}^{n}\left(k_{1}, \pm k_{2}\right) A_{t}^{n}\left(k_{1}, \pm k_{2}\right) A_{t^{\prime}}^{n}\left(k_{1}, \pm k_{2}\right)\left\{F_{t, t^{\prime}}^{-1 / 4} F_{s, s^{\prime}}^{+1 / 4}\right. \\
& \left.\quad+F_{t, t^{\prime}}^{+1 / 4} F_{s, s^{\prime}}^{-1 / 4}\right\}=1
\end{aligned}
$$

where

$$
F_{t, t^{\prime}}^{ \pm 1 / 4}=\sum_{m=0}^{\infty} \frac{\Gamma\left(\frac{m+t+t^{\prime} \pm k_{2}+1}{2}+\frac{1}{4} \pm \frac{1}{4}\right)}{m !}\left(\frac{k_{1}}{2 \omega}\right)^{m}
$$

\section{Niven approach}

Let us express solutions of the Schrödinger equation (16) in the following form: ${ }^{19}$

$$
\Psi(x, y)=e^{-\omega\left[x+\left(k_{1} / 4 \omega^{2}\right)\right]^{2}-\frac{1}{2} \omega y^{2}} y^{\frac{1}{2} \pm k_{2}} \Phi(x, y) .
$$

From Eqs. (20), (21), and (31) follows that the function $\Phi(x, y)$ is a polynomial (product of two polynomials) in terms of the variables $\left(x, y^{2}\right)$ in Cartesian coordinates and $\left(\xi^{2}, \eta^{2}\right)$ for parabolic ones. It satisfies the equation

$$
\mathcal{R} \Phi(x, y)=-2 E \Phi(x, y),
$$

where the operator $\mathcal{R}$ is

$$
\mathcal{R}=\frac{\partial^{2}}{\partial x^{2}}+\frac{\partial^{2}}{\partial y^{2}}+\left[\frac{\left(1 \pm 2 k_{2}\right)}{y}-2 \omega y\right] \frac{\partial}{\partial y}-4 \omega\left[x+\frac{k_{1}}{4 \omega^{2}}\right] \frac{\partial}{\partial x}-\omega\left(2 \pm k_{2}\right)+\frac{k_{1}^{2}}{8 \omega^{2}} .
$$

Taking into account that

$$
M k_{n q}\left(z ; k_{1}, \pm k_{2}\right)=\sum_{s=0}^{n} A_{s}^{n q}\left(k_{1}, \pm k_{2}\right) z^{s}=\prod_{\ell=1}^{n}\left(z-\alpha_{\ell}\right),
$$

where $\alpha_{\ell}, \ell=1,2, \ldots, n$ are zeros of polynomials $M k_{n q}(z)$ on the real axis $-\infty<z<\infty$, and that in parabolic coordinates 


$$
\frac{y^{2}}{\alpha}+2 x-\alpha=\frac{\left(\xi^{2}-\alpha\right)\left(\eta^{2}+\alpha\right)}{\alpha},
$$

we can choose a solution of Eq. (52) in the form

$$
\Phi(x, y)=M k_{n q_{1}}\left(\xi^{2} ; k_{1}, \pm k_{2}\right) M k_{n q_{2}}\left(-\eta^{2} ; k_{1}, \pm k_{2}\right) \cong \Pi_{\ell=1}^{n}\left(\frac{y^{2}}{\alpha_{\ell}}+2 x-\alpha_{\ell}\right) .
$$

Then from (52) it follows that the zeros $\alpha_{\ell}$ must satisfy the systems of $n$ algebraic equations

$$
\sum_{m \neq \ell}^{n} \frac{2}{\alpha_{\ell}-\alpha_{m}}+\frac{\left(1 \pm k_{2}\right)}{\alpha_{\ell}}-\omega \alpha_{\ell}=\frac{k_{1}}{2 \omega}, \quad \ell=1,2, \ldots, n
$$

and for the energy spectrum we again have a formula (22). The system of algebraic equations (57) contains $n$ sets of solutions (zeros) $\left(\alpha_{1}^{(q)}, \alpha_{2}^{(q)}, \ldots, \alpha_{n}^{(q)}\right), q=1,2, \ldots, n$ and all zeros are real. The positive zeros $\alpha_{\ell}>0$ define the nodes of wave functions for Eq. (26), whereas negative zeros $\alpha_{\ell}<0$ define the nodes of wave functions for Eq. (27).

The eigenvalues of the parabolic separation constant can be calculated in the same way via the operator equation $\Lambda \Phi(x, y)=\lambda \Phi(x, y)$ (see for details Ref. 19). A more elegant way is to use directly the differential equation (34). ${ }^{63}$ We first rewrite Eq. (34) in the form

$$
\begin{aligned}
& \left\{4 z \frac{d^{2}}{\mathrm{~d} z^{2}}+4\left[\left(1 \pm k_{2}\right)-\omega z\left(z+\frac{k_{1}}{2 \omega^{2}}\right)\right] \frac{d}{\mathrm{~d} z}+\left[4 n \omega z-\frac{k_{1}}{\omega}\left(1 \pm k_{2}\right)\right]\right\} M k_{n q}\left(z ; k_{1}, \pm k_{2}\right) \\
& =\lambda M k_{n q}\left(z ; k_{1}, \pm k_{2}\right) .
\end{aligned}
$$

Setting the wave function $M k_{n q}\left(z ; k_{1}, \pm k_{2}\right)$ in the form of (54), we arrive at the following result:

$$
\lambda_{n q}\left(k_{1}, \pm k_{2}\right)=4\left(1 \pm k_{2}\right)\left[\frac{k_{1}}{4 \omega}+\sum_{\ell=1}^{n} \frac{1}{\alpha_{\ell}^{(q)}}\right],
$$

(in case of $n=0$ the sum must be eliminated) where the quantum number $q=1,2, \ldots, n$ labels the eigenvalue of the parabolic separation constant.

\section{THE SINGULAR CIRCULAR OSCILLATOR}

The potential of the singular circular oscillator is $\left(k_{1}, k_{2}>0\right)$

$$
V_{2}(x, y)=\frac{1}{2} \omega^{2}\left(x^{2}+y^{2}\right)+\frac{1}{2}\left(\frac{k_{1}^{2}-\frac{1}{4}}{x^{2}}+\frac{k_{2}^{2}-\frac{1}{4}}{y^{2}}\right) .
$$

The corresponding Schrödinger equation separates in three different orthogonal coordinate systems: Cartesian, polar, and elliptical coordinates.

\section{A. Cartesian bases}

From the asymptotic ansatz,

$$
\Psi(x, y)=x^{\frac{1}{2} \pm k_{1}} y^{\frac{1}{2} \pm k_{2}} \exp \left[-\omega\left(x^{2}+y^{2}\right)\right] X(x) X(y)
$$

we obtain two independent and identical separation equations 


$$
\left[\frac{\partial^{2}}{\partial z_{i}^{2}}+\left(-2 \omega+\frac{1 \pm 2 k_{i}}{x_{i}^{2}}\right) x \frac{\partial}{\partial x_{i}}-\left(1 \pm 2 k_{i}\right) \omega\right] X\left(x_{i}\right)=2 \lambda_{i} X\left(x_{i}\right), \quad i=1,2
$$

where $x_{1}=x, x_{2}=y$, and $\lambda_{1}+\lambda_{2}=-E$. As in the case of the singular anisotropic oscillator we assume that the positive sign of $k_{i}$ must be taken if $k_{i}>\frac{1}{2}$ and both the positive and the negative sign must be taken if $0<k_{i}<\frac{1}{2}$.

The last equation is just that for confluent hypergeometric functions. The quantization rule gives

$$
\lambda_{i}=-\omega\left(2 n_{i} \pm k_{i}+1\right), \quad n_{i}=0,1,2, \ldots
$$

and the solution of Eq. (62) in terms of Laguerre polynomials is $X\left(x_{i}\right)=L_{n_{i}}^{ \pm k_{i}}\left(\omega x_{i}^{2}\right)$. Thus the corresponding set of orthonormal eigenfunctions which are normalized in quadrant $x>0, y>0$ (on $1 / 4)$ is

$$
\Psi_{n_{1}, n_{2}}^{\left( \pm k_{1} \pm k_{2}\right)}(x, y)=C_{n_{1}, n_{2}}^{\left( \pm k_{1}, \pm k_{2}\right)}(x)^{\frac{1}{2} \pm k_{1}}(y)^{\frac{1}{2} \pm k_{2}} e^{-(\omega / 2)\left(x^{2}+y^{2}\right)} L_{n_{1}}^{ \pm k_{1}}\left(\omega x^{2}\right) L_{n_{2}}^{ \pm k_{2}}\left(\omega y^{2}\right)
$$

where

$$
C_{n_{1}, n_{2}}^{\left( \pm k_{1} \pm k_{2}\right)}=\sqrt{\frac{\omega^{2 \pm k_{1} \pm k_{2}} n_{1} ! n_{2} !}{\Gamma\left(n_{1} \pm k_{1}+1\right) \Gamma\left(n_{2} \pm k_{2}+1\right)}} .
$$

From (63) we have

$$
E_{n}=\omega\left(2 n+2 \pm k_{1} \pm k_{2}\right),
$$

where $n=n_{1}+n_{2}=0,1,2, \ldots$ is the principal quantum number and the degree of degeneracy is $n$ +1 .

\section{B. Polar bases}

Separation of variables in the Schrödinger equation for the potential (61) in polar coordinates

$$
x=r \cos \phi, \quad y=r \sin \phi, \quad 0 \leqslant r<\infty, \quad 0 \leqslant \phi<2 \pi
$$

gives us the orthonormal solution in polynomial form

$$
\begin{aligned}
\Psi_{n_{r}, m}^{\left( \pm k_{1}, \pm k_{2}\right)}(r, \phi)= & \sqrt{\frac{2 \omega n_{r} !}{\Gamma\left(n_{r}+2 m \pm k_{1} \pm k_{2}+2\right)}}(\sqrt{\omega r})^{\left(2 m \pm k_{1} \pm k_{2}+1\right)} e^{-\omega r^{2} / 2} L_{n_{r}}^{2 q \pm k_{1} \pm k_{2}+1}\left(\omega r^{2}\right) \Phi_{m}^{\left( \pm k_{1}, \pm k_{2}\right)}(\phi) \\
& n_{r}, m=0,1,2, \ldots, \\
\Phi_{m}^{\left( \pm k_{1}, \pm k_{2}\right)}(\phi)= & \sqrt{\frac{\left(2 m \pm k_{1} \pm k_{2}+1\right) q ! \Gamma\left(q \pm k_{1} \pm k_{2}+1\right)}{2 \Gamma\left(m \pm k_{2}+1\right) \Gamma\left(m \pm k_{1}+1\right)}}(\cos \phi)^{1 / 2 \pm k_{1}}(\sin \phi)^{1 / 2 \pm k_{2}} P_{m}^{\left( \pm k_{2}, \pm k_{1}\right)} \\
& \times(\cos 2 \phi),
\end{aligned}
$$

where $P_{m}^{(\alpha, \beta)}(x)$ is a Jacobi polynomial and $E=\omega\left(2 n \pm k_{1} \pm k_{2}+2\right)$, with $n=n_{r}+m$ and with the same degree of degeneracy $(n+1)$.

Thus the quantum system (60) is exactly solvable in the Cartesian and polar systems of coordinates.

\section{Elliptic bases}

\section{Separation of variables}

Elliptic coordinates $(\nu, \mu)$ connect with Cartesian ones by $(0 \leqslant \nu<\infty, 0 \leqslant \mu<2 \pi)$ 


$$
x=\frac{D}{2} \cosh \nu \cos \mu, \quad y=\frac{D}{2} \sinh \nu \sin \mu,
$$

where $D$ is the interfocal distance. The Laplacian and volume element are

$$
\Delta=\frac{8}{D^{2}(\cosh 2 \nu-\cos 2 \mu)}\left(\frac{\partial^{2}}{\partial \nu^{2}}+\frac{\partial^{2}}{\partial \mu^{2}}\right), \quad \mathrm{d} V=\frac{D^{2}}{8}(\cosh 2 \nu-\cos 2 \mu) \mathrm{d} \nu \mathrm{d} \mu .
$$

The Schrödinger equation with (60) can be rewritten as

$$
\begin{aligned}
\frac{\partial^{2} \psi}{\partial \nu^{2}} & +\frac{\partial^{2} \psi}{\partial \mu^{2}}+\left\{\frac{D^{2} E}{4}(\cosh 2 \nu-\cos 2 \mu)-\frac{D^{4} \omega^{2}}{64}\left(\cosh ^{2} 2 \nu-\cos ^{2} 2 \mu\right)-\left[\frac{\left(k_{1}^{2}-\frac{1}{4}\right)}{\cos ^{2} \mu}+\frac{\left(k_{2}^{2}-\frac{1}{4}\right)}{\sin ^{2} \mu}\right]\right. \\
& \left.-\left[\frac{\left(k_{1}^{2}-\frac{1}{4}\right)}{\sinh ^{2} \nu}-\frac{\left(k_{2}^{2}-\frac{1}{4}\right)}{\cosh ^{2} \nu}\right]\right\} \psi=0
\end{aligned}
$$

and after the separation ansatz

$$
\psi\left(\nu, \mu ; D^{2}\right)=X\left(\nu ; D^{2}\right) Y\left(\mu ; D^{2}\right)
$$

transforms to two ordinary differential equations

$$
\begin{gathered}
\frac{d^{2} X}{\mathrm{~d} \nu^{2}}+\left[\frac{D^{2} E}{4} \cosh 2 \nu-\frac{D^{4} \omega^{2}}{64} \cosh ^{2} 2 \nu-\frac{k_{2}^{2}-\frac{1}{4}}{\sinh ^{2} \nu}+\frac{k_{1}^{2}-\frac{1}{4}}{\cosh ^{2} \nu}\right] X=-\lambda\left(D^{2}\right) X, \\
\frac{d^{2} Y}{\mathrm{~d} \mu^{2}}-\left[\frac{D^{2} E}{4} \cos 2 \mu-\frac{D^{4} \omega^{2}}{64} \cos ^{2} 2 \mu+\frac{k_{1}^{2}-\frac{1}{4}}{\cos ^{2} \mu}+\frac{k_{2}^{2}-\frac{1}{4}}{\sin ^{2} \mu}\right] Y=+\lambda\left(D^{2}\right) Y,
\end{gathered}
$$

where $\lambda$ is the elliptic separation constant. These equations can be written in the unit form

$$
\frac{d^{2} Z(\zeta)}{\mathrm{d} \zeta^{2}}+\left[\frac{D^{4} \omega^{2}}{64} \cos ^{2} 2 \zeta-\frac{D^{2} E}{4} \cos 2 \zeta-\frac{k_{1}^{2}-\frac{1}{4}}{\cos ^{2} \zeta}-\frac{k_{2}^{2}-\frac{1}{4}}{\sin ^{2} \zeta}\right] Z(\zeta)=\lambda\left(D^{2}\right) Z(\zeta),
$$

where at $\zeta \in[0,2 \pi]$ we have the equation (75) but at $\zeta \in[0, i \infty)$-Eq. (74). In other words, in the complex $\zeta$ plane the "physical regions" are only the shaded domains on the two lines $\operatorname{Im} \zeta=0$ and $\operatorname{Re} \zeta=0$.

For $k_{1,2}>\frac{1}{2}$ the centrifugal barrier is repulsive and motion takes place in only one of the quadrants, as $\zeta \in[0, \pi / 2]$, whereas for $0<k_{1,2}<\frac{1}{2}$ it takes place in the whole region $\zeta \in[0,2 \pi]$. For the particular case $k_{1}=k_{2}=\frac{1}{2}$ the equation (76) transforms to the problem of the ordinary two-dimensional oscillator and has been investigated in detail in Ref. 69. In this paper we have shown that the solution of Eq. (76) (for $k_{1}=k_{2}=1 / 2$ ) is described by Ince polynomials. ${ }^{80}$

In the case where $k_{1}$ and $k_{2}$ are integers, Eqs. (74) and (75) coincide with those that have been found via separation of variables in the Schrödinger equation for the four-dimensional isotropic oscillator in spheroidal coordinates. ${ }^{70}$

\section{Recurrence relations}

Let us now consider the equation (76). First, introducing the function $W\left(\zeta ; D^{2}\right)$ according to

$$
Z\left(\zeta ; D^{2}\right)=\exp \left[-\frac{D^{2} \omega}{16} \cos 2 \zeta\right] W\left(\zeta ; D^{2}\right)
$$

we have the equation 


$$
\frac{d^{2} W}{\mathrm{~d} \zeta^{2}}+\frac{D^{2} \omega}{4} \sin 2 \zeta \frac{\mathrm{d} W}{\mathrm{~d} \zeta}+\left[\frac{D^{2} \omega}{4} \cos 2 \zeta-\frac{D^{2} E}{2} \cos ^{2} \zeta-\frac{k_{1}^{2}-\frac{1}{4}}{\cos ^{2} \zeta}-\frac{k_{2}^{2}-\frac{1}{4}}{\sin ^{2} \zeta}-\lambda\right] W=0
$$

For $k_{1}=k_{2}=1 / 2$ this is the Ince equation. ${ }^{50}$

Next the substitution

$$
W\left(\zeta ; D^{2}\right)=(\sin \zeta)^{\frac{1}{2} \pm k_{2}}(\cos \zeta)^{\frac{1}{2} \pm k_{1}} U\left(\zeta ; D^{2}\right)
$$

yields the equation

$$
\frac{d^{2} U}{\mathrm{~d} \zeta^{2}}+\left[\left(1 \pm 2 k_{2}\right) \cot \zeta-\left(1 \pm 2 k_{1}\right) \tan \zeta+\frac{D^{2} \omega}{4} \sin 2 \zeta\right] \frac{\mathrm{d} U}{\mathrm{~d} \zeta}+\left[p \cos ^{2} \zeta-\tilde{\lambda}\right] U=0
$$

where

$$
p=\frac{D^{2}}{2}\left[\omega\left(2 \pm k_{1} \pm k_{2}\right)-E\right], \quad \tilde{\lambda}=\lambda+\frac{D^{2} \omega}{2}\left(1 \pm k_{1}\right)+\left(1 \pm k_{1} \pm k_{2}\right)^{2}-\frac{D^{2} E}{4}-\frac{D^{4} \omega^{2}}{64} .
$$

Passing to a new variable $t=\cos ^{2} \zeta$ we find

$$
t(1-t) \frac{d^{2} U}{\mathrm{~d} t^{2}}+\left\{\left(1 \pm k_{1}\right)(1-t)-\left(1 \pm k_{2}\right) t+\frac{D^{2} \omega}{4} t(t-1)\right\} \frac{d U}{\mathrm{~d} t}+\frac{1}{4}[p t-\tilde{\lambda}] U=0 .
$$

Finally, looking for the solution of the last equation in the form

$$
U\left(t ; D^{2}\right)=\sum_{s=0}^{\infty} A_{s}\left(D^{2}\right) t^{s},
$$

for coefficients $A_{s}\left(D^{2}\right)$ we have the three-term recurrence relation

$$
(s+1)\left(s+1 \pm k_{1}\right) A_{s+1}-\left[s\left(s+1 \pm k_{1} \pm k_{2}\right)+\frac{D^{2} \omega}{4} s+\frac{\tilde{\lambda}}{4}\right] A_{s}+\frac{1}{4}\left[p+D^{2} \omega(s-1)\right] A_{s-1}=0
$$

with $A_{-1}=0$ and initial condition $A_{0}=1$.

\section{Energy spectrum and separation constant}

In analogy with our asymptotic solution of the recurrence relation for the singular anisotropic operator in the parabolic basis we use continued fractions. For the minimal solution of the recurrence relations we find for $s^{-1} \ll 1$,

$$
\frac{A_{s+1}}{A_{s}} \sim \frac{D^{2} \omega}{4 s}\left(1+O\left(\frac{1}{\sqrt{s}}\right)\right) .
$$

Thus we have

$$
A_{s} \sim \frac{\left(\frac{D^{2} \omega}{4}\right)^{s}}{s !}
$$

and 


$$
U(\cos \zeta) \sim \sum \frac{\left(\frac{D^{2} \omega}{4}\right)^{k}}{k !} \cos ^{2 k} \zeta \sim \exp \left(\frac{D^{2} \omega}{8} \cos 2 \zeta\right) .
$$

Therefore we see that for this case the function $Z\left(\cos \zeta ; D^{2}\right)$ as $\zeta \rightarrow i \infty$ is not normalizable. There is a linearly independent solution of the recurrence relations, but the coefficients grow even faster. Hence it follows that the series (83) should be truncated. The condition that the series (83) be truncated gives us well-known formulas for the energy spectrum (66) and reduces the solution to polynomials,

$$
U_{n}^{\left( \pm k_{1}, \pm k_{2}\right)}\left(t ; D^{2}\right)=\sum_{s=0}^{n} A_{s}^{\left( \pm k_{1}, \pm k_{2}\right)}\left(D^{2}\right) t^{s}
$$

where now the coefficients $A_{s} \equiv A_{s}^{\left( \pm k_{1}, \pm k_{2}\right)}\left(D^{2}\right)$ satisfy the following three-term recurrent relations:

$$
(s+1)\left(s+1 \pm k_{1}\right) A_{s+1}+\beta_{s} A_{s}-\frac{D^{2} \omega}{4}(n-s+1) A_{s-1}=0, \quad s=0,1, \ldots, n
$$

with

$$
\beta_{s}=-\frac{1}{4}\left[\left(2 s+1 \pm k_{1} \pm k_{2}\right)^{2}+\frac{D^{2} \omega}{2}\left(2 s-n+4 \pm 4 k_{1}\right)-\frac{D^{2} \omega}{4}\left(2 \pm k_{1} \pm k_{2}\right)-\frac{D^{4} \omega^{2}}{64}+\lambda\left(D^{2}\right)\right]
$$

and $A_{-1}=A_{n+1}=0$.

The recurrence relations (88) become a system of $(n+1)$ linear homogeneous equations for the coefficients $A_{s}$. Equating the corresponding determinant to zero,

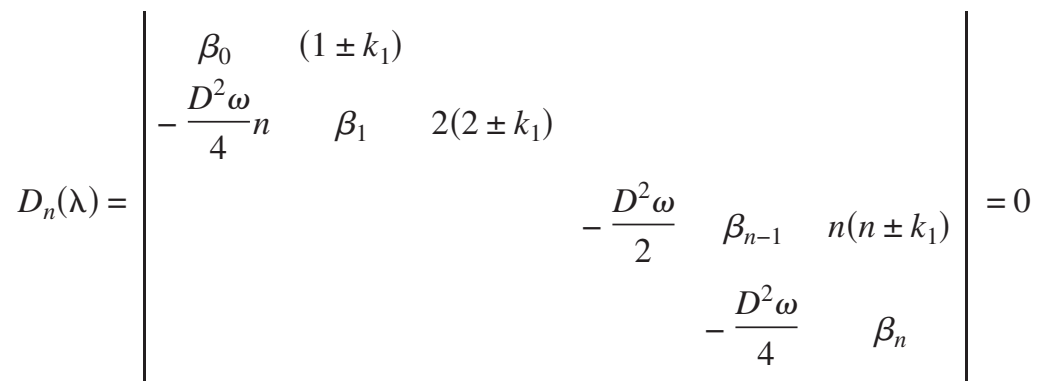

leads to the algebraic equation of degree $(n+1)$ which determines the eigenvalues of the elliptic separation constant $\lambda_{n q}^{\left( \pm k_{1}, \pm k_{2}\right)}\left(D^{2}\right)$. The quantum number $q=0,1,2, \ldots, n$ labels the $(n+1)$ roots of Eq. (90) and therefore the degree of degeneracy, as in the polar and Cartesian cases, for the $n$th energy state is $n+1$. It is also known that the corresponding enumeration of the quantum number $q$ defines the numbers of zeros of the polynomial (87), which has exactly $n$ zeros situated in the open interval $0<t<\infty$, and therefore, the elliptic separation constant $\lambda_{n q}^{\left( \pm k_{1}, \pm k_{2}\right)}\left(D^{2}\right)$ may be ordered also by the numbers of the nodes of the eigenfunction of Eq. (76).

\section{Wave functions}

The condition of finiteness of the solution of Eq. (78) allows the following polynomials:

$$
\mathcal{I}_{n q}^{\left( \pm k_{1}, \pm k_{2}\right)}\left(\zeta ; D^{2}\right)=(\sin \zeta)^{\frac{1}{2} \pm k_{2}}(\cos \zeta)^{\frac{1}{2} \pm k_{1}} \sum_{s=0}^{n} A_{s}^{\left( \pm k_{1}, \pm k_{2}\right)}\left(D^{2}\right)(\cos \zeta)^{2 s}
$$

while the corresponding solution of Eq. (76) is

$$
\mathcal{Z}_{n q}^{\left( \pm k_{1}, \pm k_{2}\right)}\left(\zeta ; D^{2}\right)=e^{-\left(D^{2} \omega / 16\right) \cos 2 \zeta} \mathcal{I}_{n q}^{\left( \pm k_{1}, \pm k_{2}\right)}\left(\zeta ; D^{2}\right) .
$$


We will denote the polynomials $\mathcal{I}_{n q}^{\left( \pm k_{1}, \pm k_{2}\right)}\left(\zeta ; D^{2}\right)$ as associated Ince polynomials. In the case of $k_{1}=k_{2}=1 / 2$ these polynomials transform to the four types of ordinary Ince polynomials, which are even or odd with respect to the changes $\zeta \rightarrow-\zeta$ and $\zeta \rightarrow \zeta+\pi{ }^{80,69}$

At $\zeta=\mu$ the wave functions (92) give us the solution of the angular equation (75), and for $\zeta=i \nu$ the solution of the radial equation (74). For each of the wave functions, radial or angular, there corresponds a definite number of zeros which can be represented by two quantum numbers $q_{1}$ and $q_{2}$, obeying the condition $q_{1}+q_{2}=n$. Then the complete elliptic wave function (73) may be written as

$$
\Psi_{n q_{1} q_{2}}^{\left( \pm k_{1}, \pm k_{2}\right)}\left(\nu, \mu ; D^{2}\right)=C_{n q_{1} q_{2}}\left( \pm k_{1}, \pm k_{2} ; D^{2}\right) \mathcal{Z}_{n q_{1}}^{\left( \pm k_{1}, \pm k_{2}\right)}\left(\mu ; D^{2}\right) \mathcal{Z}_{n q_{2}}^{\left( \pm k_{1}, \pm k_{2}\right)}\left(i \nu ; D^{2}\right),
$$

where $C_{n q_{1} q_{2}}\left( \pm k_{1}, \pm k_{2} ; D^{2}\right)$ is the normalization constant. It could be calculated from the condition

$$
\frac{D^{2}}{4} \int_{0}^{\infty} \mathrm{d} \nu \int_{0}^{\pi / 2} \mathrm{~d} \mu\left(\cosh ^{2} \nu-\cos ^{2} \mu\right) \Psi_{n q_{1} q_{2}}^{\left( \pm k_{1}, \pm k_{2}\right)^{*}}\left(\nu, \mu ; D^{2}\right) \Psi_{n q_{1} q_{2}}^{\left( \pm k_{1}, \pm k_{2}\right)}\left(\nu, \mu ; D^{2}\right)=\frac{1}{4}
$$

\section{Orthogonality relations}

The wave functions (93) as eigenfunctions of the Hamiltonians are orthogonal $n \neq n^{\prime}$,

$$
\int_{0}^{\infty} \int_{0}^{\pi / 2} \Psi_{n^{\prime} q_{1} q_{2}}^{\left( \pm k_{1} \pm k_{2}\right)^{*}}\left(\nu, \mu ; D^{2}\right) \Psi_{n q_{1} q_{2}}^{\left( \pm k_{1} \pm k_{2}\right)}\left(\nu, \mu ; D^{2}\right) \mathrm{d} V=0 .
$$

Equations (74) and (75) enable one to prove the property of double orthogonality for wave functions $\mathcal{Z}_{n q}^{\left( \pm k_{1}, \pm k_{2}\right)}\left(\zeta ; D^{2}\right)$, namely

$$
\begin{gathered}
\int_{0}^{\infty} \mathcal{Z}_{n q_{2}^{\prime}}^{\left( \pm k_{1} \pm k_{2}\right)^{*}}\left(i \nu ; D^{2}\right) \mathcal{Z}_{n q_{2}}^{\left( \pm k_{1}, \pm k_{2}\right)}\left(i \nu ; D^{2}\right) \mathrm{d} \nu=0, \\
\int_{0}^{\pi / 2} \mathcal{Z}_{n q_{1}^{\prime}}^{\left( \pm k_{1} \pm k_{2}\right)^{*}}\left(\mu ; D^{2}\right) \mathcal{Z}_{n q_{1}}^{\left( \pm k_{1} \pm k_{2}\right)}\left(\mu ; D^{2}\right) \mathrm{d} \mu=0
\end{gathered}
$$

for $q_{1} \neq q_{1}^{\prime}$ and $q_{2} \neq q_{2}^{\prime}$, and therefore when $q \neq q^{\prime}$,

$$
\int_{0}^{\infty} \mathrm{d} \nu \int_{0}^{\pi / 2} \mathrm{~d} \mu\left(\cosh ^{2} \nu-\cos ^{2} \mu\right) \Psi_{n q_{1}^{\prime} q_{2}^{\prime}}^{\left( \pm k_{1} \pm k_{2}\right)^{*}}\left(\nu, \mu ; D^{2}\right) \Psi_{n q_{1} q_{2}}^{\left( \pm k_{1} \pm k_{2}\right)}\left(\nu, \mu ; D^{2}\right)=0 .
$$

\section{THREE-DIMENSIONAL SPACE}

So far we have considered only superintegrable systems in two dimensions. To make clearer our approach and how it extends to all dimensions, we consider some three-dimensional (3D) examples.

\section{A. The harmonic oscillator}

As is very well known, the Schrödinger equation for the 3D harmonic oscillator (a superintegrable system) is exactly solvable in Cartesian coordinates. We consider it in elliptic coordinates where the separation equations are QES. We will show explicitly that the polynomial solutions of the uncoupled 3D problem can be found directly and that the results greatly simplify the determination of the polynomial solutions of the separated QES equations. In elliptic coordinates the Schrödinger equation has the form 


$$
\begin{aligned}
H= & \frac{1}{(u-v)(u-w)}\left[4 \sqrt{P(u)} \frac{\partial}{\partial u}\left(\sqrt{P(u)} \frac{\partial}{\partial u}\right)-\omega^{2}\left(u^{3}-E_{1} u^{2}\right)\right] \\
& +\frac{1}{(v-u)(v-w)}\left[4 \sqrt{P(v)} \frac{\partial}{\partial v}\left(\sqrt{P(v)} \frac{\partial}{\partial v}\right)-\omega^{2}\left(v^{3}-E_{1} v^{2}\right)\right] \\
& +\frac{1}{(v-w)(v-u)}\left[4 \sqrt{P(w)} \frac{\partial}{\partial w}\left(\sqrt{P(w)} \frac{\partial}{\partial w}\right)-\omega^{2}\left(w^{3}-E_{1} w^{2}\right)\right],
\end{aligned}
$$

where

$$
E_{1}=e_{1}+e_{2}+e_{3}, \quad P(\lambda)=\left(\lambda-e_{1}\right)\left(\lambda-e_{2}\right)\left(\lambda-e_{3}\right) .
$$

Here the elliptic coordinates are given by

$$
\begin{gathered}
x^{2}=\frac{\left(u-e_{1}\right)\left(v-e_{1}\right)\left(w-e_{1}\right)}{\left(e_{1}-e_{2}\right)\left(e_{1}-e_{3}\right)}, \quad y^{2}=\frac{\left(u-e_{2}\right)\left(v-e_{2}\right)\left(w-e_{2}\right)}{\left(e_{2}-e_{1}\right)\left(e_{2}-e_{3}\right)}, \\
z^{2}=\frac{\left(u-e_{3}\right)\left(v-e_{3}\right)\left(w-e_{3}\right)}{\left(e_{3}-e_{2}\right)\left(e_{3}-e_{1}\right)} .
\end{gathered}
$$

The separation equations that describe the solutions of $H \Psi=E \Psi$ are

$$
\left[4 \sqrt{P(\lambda)} \frac{\partial}{\partial \lambda}\left(\sqrt{P(\lambda)} \frac{\partial}{\partial \lambda}\right)-\omega^{2}\left(\lambda^{3}+\left(-E_{1}+E\right) \lambda^{2}\right)+L_{1} \lambda-L_{2}\right] \Lambda(\lambda)=0
$$

for $\lambda=u, v, w$. The operators that describe the separation constants are

$$
\begin{aligned}
L_{1}= & \frac{v w}{(u-v)(u-w)}\left[4 \sqrt{P(u)} \frac{\partial}{\partial u}\left(\sqrt{P(u)} \frac{\partial}{\partial u}\right)-\omega^{2}\left(u^{3}-E_{1} u^{2}\right)\right] \\
& +\frac{u w}{(v-u)(v-w)}\left[4 \sqrt{P(v)} \frac{\partial}{\partial v}\left(\sqrt{P(v)} \frac{\partial}{\partial v}\right)-\omega^{2}\left(v^{3}-E_{1} v^{2}\right)\right] \\
& +\frac{u v}{(w-u)(w-v)}\left[4 \sqrt{P(w)} \frac{\partial}{\partial w}\left(\sqrt{P(w)} \frac{\partial}{\partial w}\right)-\omega^{2}\left(w^{3}-E_{1} w^{2}\right)\right],
\end{aligned}
$$

and

$$
\begin{aligned}
L_{2}= & \frac{v+w}{(u-v)(u-w)}\left[4 \sqrt{P(u)} \frac{\partial}{\partial u}\left(\sqrt{P(u)} \frac{\partial}{\partial u}\right)-\omega^{2}\left(u^{3}-E_{1} u^{2}\right)\right] \\
& +\frac{u+w}{(v-u)(v-w)}\left[4 \sqrt{P(v)} \frac{\partial}{\partial v}\left(\sqrt{P(v)} \frac{\partial}{\partial v}\right)-\omega^{2}\left(v^{3}-E_{1} v^{2}\right)\right] \\
& +\frac{u+v}{(w-u)(w-v)}\left[4 \sqrt{P(w)} \frac{\partial}{\partial w}\left(\sqrt{P(w)} \frac{\partial}{\partial w}\right)-\omega^{2}\left(w^{3}-E_{1} w^{2}\right)\right] .
\end{aligned}
$$

In order to find square integrable solutions to this problem it is natural to remove an exponential factor according to

$$
\Psi(u, v, w)=\exp \left(-\frac{\omega}{2}(u+v+w)\right) \Phi(u, v, w) .
$$

Then there are polynomial solutions for $\Phi(u, v, w)$ of the form 


$$
\Phi(u, v, w)=\prod_{j=1}^{r}\left(u-\theta_{j}\right)\left(v-\theta_{j}\right)\left(w-\theta_{j}\right) .
$$

The zeros of the polynomials satisfy the relations

$$
-4 \omega+\sum_{i \neq j} \frac{4}{\theta_{i}-\theta_{j}}+\sum_{s=1}^{3} \frac{1}{\theta_{j}-e_{s}}=0 .
$$
form

It follows that the eigenvalues $E$ and $\ell_{1}, \ell_{2}$ of the operators $L_{1}$ and $L_{2}$ can be expressed in the

$$
\begin{gathered}
\ell_{1}=-4 r^{2} E_{1}+2(3+4 r) \sum_{j=1}^{r} \theta_{j}+\left[-(1+4 r) E_{2}+4 E_{1} \sum_{j=1}^{r} \theta_{j}-\sum_{j=1}^{r} \theta_{j}^{2}\right] \omega-E_{3} \omega^{2}, \\
\ell_{2}=-2 r(2 r+1)-2(2 r+1) E_{1} \omega+4 \omega \sum_{j=1}^{r} \theta_{i}-E_{2} \omega^{2},
\end{gathered}
$$

where $E_{2}=e_{1} e_{2}+e_{2} e_{3}+e_{1} e_{3}$ and $E_{3}=e_{1} e_{2} e_{3}$. Because of the relations among the zeros $\theta_{j}$ there are also alternative expressions available for these eigenvalues. We now turn our attention to calculating the eigenvalues. Let us first consider the special case $r=1$. If we choose a basis of functions of $u, v$, and $w F_{0}=1, F_{1}=u+v+w, F_{2}=u v+u w+v w$, and $F_{3}=u v w$ then we can find solutions

$$
\Phi(u, v, w)=a_{0} F_{0}+a_{1} F_{1}+a_{2} F_{2}+a_{3} F_{3} .
$$

If we look for eigenfunctions for the operator $L_{1}$ that correspond to this form we obtain the conditions

$$
\begin{gathered}
\left(2 E_{3} \omega^{2}+5 E_{2} \omega+4 E_{1}+\ell_{1}\right) a_{3}+\left(4 E_{1} \omega+6\right) a_{2}+4 \omega a_{1}=0, \\
\left(-2 E_{2}-4 E_{3} \omega\right) a_{3}+\left(\ell_{1}+\omega E_{2}+\omega^{2} E_{3}\right) a_{2}=0, \\
\left(-2 E_{2}-4 E_{3} \omega\right) a_{2}+\left(\ell_{1}+\omega E_{2}+\omega^{2} E_{3}\right) a_{1}=0, \quad\left(-2 E_{2}-4 E_{3} \omega\right) a_{1}+\left(\ell_{1}+\omega E_{2}+\omega^{2} E_{3}\right) a_{0}=0 .
\end{gathered}
$$

For these equations to have a nontrivial solution the corresponding determanental condition must hold viz.

$$
\begin{aligned}
\left(\ell_{1}\right. & \left.+\omega E_{2}+\omega^{2} E_{3}\right)\left(\ell_{1}^{3}+\left(4 E_{1}+7 E_{2} \omega+3 E_{3} \omega^{2}\right) \ell_{1}^{2}+\left(14 E_{3} E_{2} \omega^{3}+\left(24 E_{1} E_{3}+11 E_{2}^{2}\right) \omega^{2}\right.\right. \\
& \left.+\left(16 E_{1} E_{2}+24 E_{3}\right) \omega+12 E_{2}\right) \ell_{1}+E_{3}\left(20 E_{1} E_{3}+11 E_{2}^{2}\right) \omega^{4}+\left(5 E_{2}^{3}+188 E_{3}^{2}+32 E_{1} E_{2} E_{3}\right) \omega^{3} \\
& \left.+4 E_{2}\left(6 E_{1} E_{2}-14 E_{3}\right) \omega^{2}+28 E_{2} \omega\right)=0 .
\end{aligned}
$$

For the operator $L_{2}$ the corresponding relations among the $a_{i}$ are

$$
\begin{gathered}
\left(6+\ell_{2}+E_{2} \omega^{2}+6 \omega E_{1}\right) a_{3}+4 \omega a_{2}=0, \quad\left(6+\ell_{2}+E_{1} \omega+E_{2} \omega^{2}\right) a_{2}+4 \omega a_{3}=0 \\
-\left(2 E_{2}-4 E_{3} \omega\right) a_{3}+\left(-4 E_{2} \omega+4 E_{1}\right) a_{2}+\left(E_{2} \omega^{2}+2 \omega E_{1}+\ell_{2}\right) a_{1}=0 \\
-\left(2 E_{2}-4 E_{3} \omega\right) a_{2}+\left(-4 E_{1}+4 E_{2} \omega\right) a_{1}+\left(\ell_{2}+2 E_{1} \omega+E_{2} \omega^{2}\right) a_{0}=0
\end{gathered}
$$

with the determinant condition 


$$
\begin{aligned}
\left(\ell_{2}\right. & \left.+2 \omega E_{1}+\omega^{2} E_{2}\right)\left(\ell_{2}^{3}+\left(14 \omega E_{1}+3 \omega^{2} E_{2}\right) \ell_{2}^{2}+\left(3 \omega^{4} E_{2}^{2}+28 \omega^{3} E_{1} E_{2}+\omega^{2}\left(60 E_{1}^{2}+40 E_{2}\right)\right.\right. \\
& \left.+112 \omega E_{1}+36\right) \ell_{2}+\omega^{6} E_{2}^{3}+14 \omega^{5} E_{1} E_{2}^{2}+4 \omega^{4} E_{2}\left(15 E_{1}^{2}+7 E_{2}\right)+\omega^{3}\left(72 E_{1}^{3}+208 E_{1} E_{2}-343 E_{3}\right) \\
& \left.+\omega^{2}\left(240 E_{1}^{2}+100 E_{2}\right)+168 \omega E_{1}\right)=0 .
\end{aligned}
$$

This illustrates clearly that our method gives the eigenvalues of $L_{1}$ and $L_{2}$ as solutions of polynomial equations. If we substitute in this way into the Schrödinger equation itself then we obtain the conditions

$$
\begin{gathered}
(E+7 \omega) a_{j}=0, \quad j=1,2,3, \\
\left(2 E_{2}+4 E_{3} \omega\right) a_{3}+4\left(E_{1}+\omega E_{2}\right) a_{2}+2\left(3+2 \omega E_{1}\right) a_{1}-(E+3 \omega) a_{0}=0
\end{gathered}
$$

yielding the two eigenvalues $-7 \omega$ and $-3 \omega$ for $E$. This method has obvious extensions to $r$ $=2, \ldots$.

Note that if we look for polynomial solution of the separation equations then we obtain different equations. In particular if we look for solutions of the form $\Lambda(\lambda)=\exp [-(\omega / 2) \lambda](\lambda-c)$ in the separation equation

$$
\sqrt{P(\lambda)} \frac{\partial}{\partial \lambda}\left(\sqrt{P(\lambda)} \frac{\partial}{\partial \lambda} \Lambda(\lambda)\right)+\left(\omega^{2}\left(-\lambda^{3}+\left(E_{1}-E\right) \lambda^{2}\right)+\ell_{2} \lambda-\ell_{1}\right) \Lambda(\lambda)=0
$$

we obtain the relations

$$
\begin{aligned}
& \quad \ell_{2}^{2}+\ell_{2}\left(6+8 \omega E_{1}+2 \omega^{2} E_{2}\right)+4 \omega \ell_{1}+\omega^{4} E_{2}^{2}+\omega^{3}\left(8 E_{1} E_{2}+4 E_{3}\right)+\omega^{2}\left(12 E_{1}^{2}+26 E_{2}\right)+28 \omega=0, \\
& \ell_{1} \ell_{2}+\omega\left(E_{2}+E_{3} \omega\right) \ell_{2}+\omega\left(6 E_{1}+\omega E_{2}\right) \ell_{1}+E_{2} E_{3} \omega^{4}+\omega^{3}\left(6 E_{1} E_{3}+E_{2}^{2}\right)+\omega^{2}\left(6 E_{1} E_{2}+22 E_{3}\right)+14 E_{2} \omega \\
& \quad=0
\end{aligned}
$$

where

$$
c=\left(\left(12+2 \ell_{2}\right)+6 \omega E_{1}+\omega^{2} E_{2}\right) / 4 \omega .
$$

If we were to pursue this approach further then we would obtain more complicated relations among the $\ell_{1}$ and $\ell_{2}$ which could be uncoupled to produce the individual equations for $\ell_{1}$ and $\ell_{2}$, respectively. This example shows clearly how study of the full 3D superintegrable system yields results for solutions of the separation equations that could not easily be obtained from a direct study of the separation equations themselves.

\section{B. Ushveridze's separation of variables example}

A critical further example is that studied by Ushveridze on p. 115 of Ref. 63. He takes two copies of an ordinary differential QES problem (polynomial potential of order 10) and combines them to form a single 2D partial differential equation from which the original ordinary differential equations can be obtained by separation of variables. However, the partial differential equation that he obtains is merely separable, not multiseparable. In particular it is not superintegrable. Here we show the increased insight and greater simplicity obtained by using three copies of the QES problem to form a 3D superintegrable system. We proceed as follows. Consider the Schrödinger equation $H \Psi=E \Psi$ where 


$$
\begin{aligned}
H= & \frac{1}{\left(u^{2}-v^{2}\right)\left(u^{2}-w^{2}\right)}\left[\frac{\partial^{2}}{\partial u^{2}}-36 k_{1}^{2} u^{10}-48 k_{1} k_{2} u^{8}-8\left(2 k_{2}^{2}+3 k_{1} k_{3}\right) u^{6}+\frac{p(1-p)}{u^{2}}\right] \\
& +\frac{1}{\left(v^{2}-u^{2}\right)\left(v^{2}-w^{2}\right)}\left[\frac{\partial^{2}}{\partial v^{2}}-36 k_{1}^{2} v^{10}-48 k_{1} k_{2} v^{8}-8\left(2 k_{2}^{2}+3 k_{1} k_{3}\right) v^{6}+\frac{p(1-p)}{v^{2}}\right] \\
& +\frac{1}{\left(w^{2}-v^{2}\right)\left(w^{2}-u^{2}\right)}\left[\frac{\partial^{2}}{\partial w^{2}}-36 k_{1}^{2} w^{10}-48 k_{1} k_{2} w^{8}-8\left(2 k_{2}^{2}+3 k_{1} k_{3}\right) w^{6}+\frac{p(1-p)}{w^{2}}\right] .
\end{aligned}
$$

This equation is clearly separable in the $u, v, w$ coordinates. Passing to Cartesian coordinates $z$ $=i u v w$ and

$$
x+i y=\frac{1}{2}\left(u^{2} v^{2}+u^{2} w^{2}+v^{2} w^{2}\right)-\frac{1}{4}\left(u^{4}+v^{4}+w^{4}\right), \quad x-i y=\frac{1}{2}\left(u^{2}+v^{2}+w^{2}\right),
$$

we can recognize the Hamiltonian operator in the form

$$
\begin{aligned}
H= & \frac{\partial^{2}}{\partial x^{2}}+\frac{\partial^{2}}{\partial y^{2}}+\frac{\partial^{2}}{\partial z^{2}}+36 k_{1}^{2}\left(2(x-i y)^{3}-4\left(x^{2}+y^{2}\right)-z^{2}\right)+48 k_{1} k_{2}\left(3(x-i y)^{2}-(x+i y)\right) \\
& -16\left(2 k_{2}^{2}+3 k_{1} k_{3}\right)(x+i y)-\frac{p(p-1)}{z^{2}} .
\end{aligned}
$$

This in turn can be recognized as essentially the complex Euclidean space superintegrable system with nondegenerate potential

$$
V=\alpha\left(z^{2}-2(x-i y)^{3}+4\left(x^{2}+y^{2}\right)\right)+\beta\left(2(x+i y)-3(x-i y)^{2} 2\right)+\gamma(x+i y)+\frac{\delta}{z^{2}},
$$

in which the six basis second-order symmetry operators can be taken in the form

$$
\begin{gathered}
H=\partial_{x}^{2}+\partial_{y}^{2}+\partial_{z}^{2}+V, \\
S_{1}=\left(\partial_{x}-i \partial_{y}\right) 2+f_{1}, \quad S_{2}=\partial_{z}^{2}+f_{2}, \quad S_{3}=\left\{\partial_{z}, J_{2}+i J_{1}\right\}+f_{3}, \\
S_{4}=\frac{1}{2}\left\{J_{3}, \partial_{x}-i \partial_{y}\right\}-\frac{i}{4}\left(\partial_{x}+i \partial_{y}\right)^{2}+f_{4}, \quad S_{5}=\left(J_{2}+i J_{1}\right)^{2}+2 i\left\{\partial_{z}, J_{1}\right\}+f_{5},
\end{gathered}
$$

where $\{A, B\}=A B+B A$, the $J_{i}$ are the angular momentum operators, e.g., $J_{3}=x \partial_{y}-y \partial_{x}$, and the $f_{i}$ are appropriate functions. There is a quadratic algebra generated by these symmetries. This is a direct consequence of the observation that this potential is an example of a nondegenerate potential in three dimensions. 7,8

The separation equations for the Schrödinger equation have the form

$$
\left[\frac{\partial^{2}}{\partial \lambda^{2}}-36 k_{1}^{2} \lambda^{10}-48 k_{1} k_{2} \lambda^{8}-8\left(2 k_{2}^{2}+3 k_{1} k_{3}\right) \lambda^{6}+\frac{p(1-p)}{\lambda^{2}}+E \lambda^{4}+\ell_{2} \lambda^{2}+\ell_{3}\right] \Lambda(\lambda)=0,
$$

essentially, Ushveridze's 1D QES problem. The operators with the separation constants as eigenvalues are 


$$
\begin{aligned}
L_{2}= & \frac{v^{2}+w^{2}}{\left(u^{2}-v^{2}\right)\left(u^{2}-w^{2}\right)}\left[\frac{\partial^{2}}{\partial u^{2}}-36 k_{1}^{2} u^{10}-48 k_{1} k_{2} u^{8}-8\left(2 k_{2}^{2}+3 k_{1} k_{3}\right) u^{6}+\frac{p(1-p)}{u^{2}}\right] \\
& +\frac{u^{2}+w^{2}}{\left(v^{2}-u^{2}\right)\left(v^{2}-w^{2}\right)}\left[\frac{\partial^{2}}{\partial v^{2}}-36 k_{1}^{2} v^{10}-48 k_{1} k_{2} v^{8}-8\left(2 k_{2}^{2}+3 k_{1} k_{3}\right) v^{6}+\frac{p(1-p)}{v^{2}}\right] \\
& +\frac{u^{2}+v^{2}}{\left(w^{2}-v^{2}\right)\left(w^{2}-u^{2}\right)}\left[\frac{\partial^{2}}{\partial w^{2}}-36 k_{1}^{2} w^{10}-48 k_{1} k_{2} w^{8}-8\left(2 k_{2}^{2}+3 k_{1} k_{3}\right) w^{6}+\frac{p(1-p)}{w^{2}}\right]
\end{aligned}
$$

and

$$
\begin{aligned}
L_{3}= & \frac{v^{2} w^{2}}{\left(u^{2}-v^{2}\right)\left(u^{2}-w^{2}\right)}\left[\frac{\partial^{2}}{\partial u^{2}}-36 k_{1}^{2} u^{10}-48 k_{1} k_{2} u^{8}-8\left(2 k_{2}^{2}+3 k_{1} k_{3}\right) u^{6}+\frac{p(1-p)}{u^{2}}\right] \\
& +\frac{u^{2} w^{2}}{\left(v^{2}-u^{2}\right)\left(v^{2}-w^{2}\right)}\left[\frac{\partial^{2}}{\partial v^{2}}-36 k_{1}^{2} v^{10}-48 k_{1} k_{2} v^{8}-8\left(2 k_{2}^{2}+3 k_{1} k_{3}\right) v^{6}+\frac{p(1-p)}{v^{2}}\right] \\
& +\frac{u^{2} v^{2}}{\left(w^{2}-v^{2}\right)\left(w^{2}-u^{2}\right)}\left[\frac{\partial^{2}}{\partial w^{2}}-36 k_{1}^{2} w^{10}-48 k_{1} k_{2} w^{8}-8\left(2 k_{2}^{2}+3 k_{1} k_{3}\right) w^{6}+\frac{p(1-p)}{w^{2}}\right] .
\end{aligned}
$$

In searching for finite solutions of $H \Psi=E \Psi$ we write

$$
\Psi(u, v, w)=\exp \left(k_{1}\left(u^{6}+v^{6}+w^{6}\right)+k_{2}\left(u^{4}+v^{4}+w^{4}\right)+k_{3}\left(u^{2}+v^{2}+w^{2}\right)\right)(u v w)^{p} \Phi(u, v, w),
$$

where

$$
\Phi(u, v, w)=\prod_{j=1}^{r}\left(u^{2}-\theta_{j}\right)\left(v^{2}-\theta_{j}\right)\left(w^{2}-\theta_{j}\right) .
$$

The zeros of the polynomials satisfy the relations

$$
\frac{2 r+1}{2 \theta_{i}}-12 k_{1} \theta_{i}^{2}-4 k_{2} \theta_{i}-k_{3}+\sum_{j \neq i} \frac{1}{\theta_{i}-\theta_{j}}=0 .
$$

Solving these equations we see that the eigenvalues of the operators $H, L_{2}$, and $L_{3}$ have the form

$$
\begin{gathered}
E=-(30+24 r+12 p) k_{1}-16 k_{2} k_{3}, \quad \ell_{2}=-4 k_{3}^{2}-(12+16 r) k_{2}-24 k_{1} \sum_{j=1}^{r} \theta_{j}, \\
\ell_{3}=-(2+8 r+4 p) k_{3}-16 k_{2} \sum_{j=1}^{r} \theta_{j}-24 k_{1} \sum_{j=1}^{r} \theta_{j}^{2} .
\end{gathered}
$$

Because of the relations among the zeros there are many other expressions for these eigenvalues. If we look for solutions of the form

$$
\Phi(u, v, w)=a_{0} G_{0}+a_{1} G_{1}+a_{2} G_{2}+a_{3} G_{3},
$$

where $G_{0}=1, G_{1}=u^{2}+v^{2}+w^{2}, G_{2}=u^{2} v^{2}+u^{2} w^{2}+v^{2} w^{2}$, and $G_{3}=u^{2} v^{2} w^{2}$, i.e., second-order polynomial solutions, and substitute this expression into the eigenvalue equations, we obtain the following polynomial equations for the eigenvalues:

$$
\left(E+k_{1}(54+12 p)\right)^{3}\left(E+k_{1}(30+12 p)\right)=0,
$$




$$
\begin{gathered}
\left(\ell_{2}+2 k_{2}(4 p+1)+4 k_{3}^{2}\right)\left(\ell_{2}^{3}+\left(12 k_{3}^{2}+(68+24 p) k_{2}\right) \ell_{2}^{2}\right. \\
+\left(192 k_{1} k_{3}+16(2 p+7)(6 p+13) k_{2}^{2}+32 k_{3}^{2} k_{2}(6 p+17)+48 k_{3}^{4}\right) \ell_{2} \\
+64(17+6 p) k_{2} k_{3}^{4}+768 k_{1} k_{3}^{3}+64(2 p+7)(6 p+13) k_{2}^{2} k_{3}^{2}+768(2 p+7) k_{1} k_{2} k_{3} \\
+64(2 p+3)(2 p+7)^{2} k_{3}^{2}-1152(2 p+1) k_{1}^{2}=0, \\
\left(\ell_{3}+2 k_{3}(p+1)\right)\left(\ell_{3}^{3}+2(6 p+7) k_{3} \ell_{3}^{2}+4(6 p+11)(1+2 p) k_{3}^{2} \ell_{3}+8(1+2 p)^{2}\left(12 k_{1}+2 p k_{3}^{2}+5 k_{3}^{3}\right)\right) \\
=0 .
\end{gathered}
$$

On the other hand, if we study the separation equations individually and look for a solution of the form

$$
\Lambda(\lambda)=\exp \left(k_{1} \lambda^{6}+k_{2} \lambda^{4}+k_{3} \lambda^{2}\right) \lambda^{p}(\lambda-c)
$$

in the above separation equation then we obtain different relations

$$
\begin{aligned}
& \ell_{2}^{2}+\left(4 k_{2}(10+4 p)+8 k_{3}^{2}\right) \ell_{2}+24 k_{1} \ell_{3}+16 k_{3}^{4}+8\left(4 k_{2} k_{3}^{2}+6 k_{1} k_{3}\right)(5+2 p)+16 k_{2}^{2}(2 p+7)(2 p+3)=0 \\
& \quad \ell_{2} \ell_{3}+2(2 p+1) \ell_{2}+\left(4 k_{2}(2 p+7)+4 k_{3}^{2}\right) \ell_{3}+48 k_{1}(1+2 p)+8 k_{2} k_{3}(2 p+7)(2 p+1)+8(1+2 p) k_{3}^{3} \\
& \quad=0
\end{aligned}
$$

where

$$
c=-\frac{1}{24 k_{1}}\left(4 k_{2}(p+7)+4 k_{3}^{3}+\ell_{2}\right) .
$$

The above computation extends in an obvious manner to the computation of polynomial solutions of any order. There is a clear relationship with Ushveridze's equation on p. 115 of Ref. 63 through the correspondence $a=6 k_{1}, b=4 k_{2}, c=2 k_{3}$, and $s=(2 p+1) / 4$.

We now look for solutions determined by other second-order constants of the motion and corresponding (possibly separable) coordinate systems. First consider our basic equation $H \Psi$ $=E \Psi$ written in terms of different coordinates $\xi=x+i y, \eta=x-i y, z$. We can find nonseparable solutions in these coordinates of the form

$$
\begin{aligned}
\Psi= & \exp \left(2 \sqrt{ }\left[\lambda+\left(k_{2}+3 k_{1} \xi\right)^{2}\right] \eta\right. \\
& \left.\times \int\left[-\frac{3 k_{1}\left(k_{2}+3 k_{1} \xi\right)}{\left[\lambda+\left(k_{2}+3 k_{1} \xi\right)^{2}\right]}+\frac{\xi\left(-9 k_{1}^{2} \xi^{2}+9 k_{1} k_{2} \xi+6 k_{1} k_{2}+4 k_{2}^{2}\right)+E}{\sqrt{ }\left(\lambda+\left(k_{2}+3 k_{1} \xi\right)^{2}\right)}\right] \mathrm{d} \xi\right) \\
& \times \exp \left(-3 k_{1} z^{2}\right) z^{p} L_{n}^{p+\frac{1}{2}}\left(6 k_{1} z^{2}\right),
\end{aligned}
$$

where

$$
E=E-6 k_{1}(4 n+2 p+1)
$$

and $L_{m}^{\alpha}(t)$ is a Laguerre polynomial. It is clear that the above $\xi$ integral can be calculated in terms of elementary functions but we prefer the form given as it is more compact. This possibility for an explicit solution comes about from the existence of a symmetry of the form $p_{\eta}^{2}+f$.

If we choose new separable coordinates $u, v, z$ defined by 


$$
x+i y=-\frac{1}{2}(u-v)^{2}, \quad x-i y=u+v
$$

then the Schrödinger equation has the separable form

$$
\begin{aligned}
H \Psi= & {\left[\frac { 1 } { u - v } \left[\left(\frac{\partial}{\partial u^{2}}-144 k_{1}^{2} u^{4}-96 k_{1} k_{2} u^{3}+16\left(2 k_{2}^{2}+3 k_{1} k_{3}\right) u^{2}\right)\right.\right.} \\
& \left.\left.-\left(\frac{\partial}{\partial v^{2}}-144 k_{1}^{2} v^{4}-96 k_{1} k_{2} v^{3}+16\left(2 k_{2}^{2}+3 k_{1} k_{3}\right) v^{2}\right)\right]+\frac{\partial^{2}}{\partial z^{2}}-36 k_{1}^{2} z^{2}+\frac{p(1-p)}{z^{2}}\right] \Psi=E \Psi .
\end{aligned}
$$

The symmetry operator $L$ associated with separation in these coordinates is of the form

$$
\begin{aligned}
L \Psi= & {\left[\frac { 1 } { u - v } \left[v\left(\frac{\partial}{\partial u^{2}}-144 k_{1}^{2} u^{4}-96 k_{1} k_{2} u^{3}+16\left(2 k_{2}^{2}+3 k_{1} k_{3}\right) u^{2}\right)\right.\right.} \\
& \left.-u\left(\frac{\partial}{\partial v^{2}}-144 k_{1}^{2} v^{4}-96 k_{1} k_{2} v^{3}+16\left(2 k_{2}^{2}+3 k_{1} k_{3}\right) v^{2}\right)\right] \Psi .
\end{aligned}
$$

Searching for finite solutions using these coordinates, we see that they can be taken in the form

$$
\begin{aligned}
\Psi= & \exp \left(4 k_{1}\left(u^{3}+v^{3}\right)+2 k_{2}\left(u^{2}+v^{2}\right)-2\left(\frac{k_{2}^{2}}{k_{1}}+k_{3}\right)(u+v)\right) \Pi_{i=0}^{r}\left[\left(u-\theta_{i}\right)\left(v-\theta_{i}\right)\right] \\
& \times \exp \left(-3 k_{1} z^{2}\right) z^{p} L_{n}^{p+\frac{1}{2}}\left(6 k_{1} z^{2}\right) .
\end{aligned}
$$

For solutions of this kind these zeros satisfy

$$
-2 k_{1} k_{3}-2 k_{2}^{2}+4 k_{1} k_{2} \sum_{i=1}^{r} \theta_{i}+12 k_{1}^{2} \sum_{i=1}^{r} \theta_{i}^{2}+\sum_{j \neq i} \frac{k_{1}}{\theta_{i}-\theta_{j}}=0 .
$$

The eigenvalues of $L$ and $H^{\prime}$ have the form

$$
\lambda_{r}=-4 \frac{k_{2}^{4}}{k_{1}^{2}}-8 \frac{k_{2}^{2} k_{3}}{k_{1}}-4 k_{3}^{2}-4(1+2 r) k_{2}-24 \sum_{i=1}^{r} \theta_{i}, \quad E_{r}=16 k_{2} k_{3}+16 \frac{k_{2}^{3}}{k_{1}}-24(r+1) k_{1},
$$

and $E=E_{r}+6 k_{1}(4 n+2 p+1)$.

It is clear that we can find solutions of the form given above but with a choice of polynomial, say $c_{1} u v+c_{2}(u+v)+c_{3}$ for illustration. The resulting polynomial equation for the eigenvalues of $L$ is

$$
\begin{gathered}
\left(\lambda k_{1}^{2}+4 k_{1}^{2} k_{3}^{2}+4 k_{2} k_{1}^{2}+4 k_{2}^{4}+8 k_{1} k_{3} k_{2}^{2}\right)\left(k_{1}^{4} \lambda^{2}+8 k_{1}^{2}\left(2 k_{2} k_{1}^{2}+k_{3}^{2} k_{1}^{2}+2 k_{1} k_{3} k_{1}^{2}+k_{2}^{4}\right) \lambda\right) \\
-96 k_{3} k_{1}^{5}-48 k_{2}^{2} k_{1}^{4}+64 k_{3}^{2} k_{1}^{4} k_{2}+16 k_{3}^{4} k_{1}^{4}+128 k_{2}^{3} k_{3} k_{1}^{3}+64 k_{3}^{3} k_{1}^{3} k_{2}^{2}+64 k_{2}^{5} k_{1}^{2}+96 k_{1}^{2} k_{2}^{4} k_{3}^{2} \\
+64 k_{2}^{6} k_{1} k_{3}+16 k_{2}^{8}=0 .
\end{gathered}
$$

These are finite solutions, clearly different from those given previously. The above analysis can be extended in an obvious manner to yield polynomial solutions of any order.

Note that for this last coordinate system we have given an example of a QES problem with a quartic potential, something hitherto not known to be possible (as mentioned in Ushveridze's book). Indeed the separation equations have the form 


$$
\begin{aligned}
& \left(\frac{\partial^{2}}{\partial \ell^{2}}-144 k_{1}^{2} \ell^{4}-96 k_{1} k_{2} \ell^{3}+16\left(2 k_{2}^{2}+3 k_{1} k_{3}\right)\right) \ell^{2} \\
& \quad-\left\{16 k_{2} k_{3}+16 \frac{k_{3}^{2}}{k_{1}}+6 k_{1}(4 n-4 r+2 p-3) \ell+\lambda_{r}\right\} \Lambda(\ell)=0,
\end{aligned}
$$

where $\Lambda=U, V$ and $\ell=u, v$. There are typically $r+1$ solutions

$$
\Lambda(\ell)=\exp \left(4 k_{1} \ell^{3}+2 k_{2} \ell^{2}-2\left(\frac{k_{2}^{2}}{k_{1}}+k_{3}\right) \ell\right) \Pi_{i=0}^{r}\left(\ell-\theta_{i}\right)
$$

of this equation, with corresponding eigenvalues $\lambda_{r}^{(s)}, s=1, \ldots, r+1$. It is clear from our definition of QES that if we look for series solutions then the recurrence relations involved will contain more than three terms. The analysis then proceeds in analogy with what has been demonstrated for the case of three terms and the requirement of polynomial solutions (to within a factor) is a consequence of the solutions generated in this manner being well behaved at the regular singular points.

It is clear that in higher dimensions there are many examples which generalize the examples occurring in Ref. 63. The utility of the use of partial differential operators, rather than ordinary differential operators, is evident. Finally, we note in the superintegrable example presented here, though our system is multiseparable there are no separable coordinates in which the separated equations are each exactly solvable.

\section{CONCLUSIONS AND SUMMARY}

We have demonstrated that solutions of the Schrödinger equation for the potential $V_{1}$ may be constructed via separation of variables in two different ways. Using Cartesian coordinates we arrive at two independent exactly solvable equations (17) and (18), each of them representing a one-dimensional nonparametric spectral problem where the Cartesian separation constants $\lambda_{i}$ play the role of energy. To obtain solutions in the form of Laguerre and Hermite polynomials, both separation constants are quantized and as a result the energy spectrum for the two-dimensional Schrödinger equation is obtained. For the second separable system which uses parabolic coordinates the solution method is more complex. We have shown that the separation procedure reduces to an ordinary differential equation for real and imaginary variables. It has been proven that the requirement of convergence for solutions of Eq. (30) at the singular points $\mu= \pm \infty$ and $\mu=i \infty$ leads to only polynomial solutions (42) with the restriction for the energy spectrum $E$ in the form (22) and for a fixed energy (or quantum number $n$ ) gives the spectrum of the separation constant as the root of an $n$ th-degree polynomial equation. In contrast to the solution in Cartesian coordinates the coefficients of the polynomial solutions satisfy three-term recurrence relations and cannot be written in explicit form in general. For this reason we refer to the equation (30) as quasiexactly solvable.

On the other hand, the substitution of the formula for the energy spectrum into Eq. (30) gives rise to the equation

$$
\left[-\frac{d^{2}}{\mathrm{~d} \mu^{2}}+\left(\omega^{2} \mu^{6}+k_{1} \mu^{4}+\left[\frac{k_{1}^{2}}{4 \omega^{2}}-\omega\left(4 n+4 \pm 2 k_{2}\right)\right] \mu^{2}+\frac{k_{2}^{2}-\frac{1}{4}}{\mu^{2}}\right)\right] Z_{n}(\mu)=\lambda Z_{n}(\mu)
$$

which on the real axis completely coincides for $k_{1}=4 \beta \omega^{2}$ and $1 \pm k_{2}=2 \delta$, with the one-dimensional spectral problem (10), and is called a quasiexactly solvable problem. Now it is easy to understand the origins of the occurrence of quasiexactly solvable systems. The requirement of convergence just in real space (which is possible to determine following Ref. 37 as the dimensional reduction) in the vicinity of singular points $\mu= \pm \infty$ requires that there are polynomial solutions of the form (41). We also can shed light on the mystery of the zeros of the polynomial $P_{n}\left(x^{2}\right)$. Indeed, the substitution of the wave function (11) into the Schrödinger equation with potential (10) leads to 
the differential equation for polynomial $P_{n}\left(x^{2}\right)$ in the same form as Eq. (58) (in variable $x^{2}=z$ ), but with the difference that the physical region of Eq. (58) is the whole real axis $z \in(-\infty, \infty)$, and therefore all zeros (for positive and negative $x^{2}$ ) of $P_{n}\left(x^{2}\right)$ correspond to the zeros of twodimensional eigenfunction of singular anisotropic oscillator in parabolic coordinates.

The situation is repeated in the case of the second potential (60). We have determined that the separation of variables in two-dimensional elliptic coordinates leads to a Schrödinger type equation (76) in the complex plane and the requirement of convergence at the point $\zeta=0,2 \pi$ and $\zeta$ $=i \infty$ requires polynomial solutions and defines the energy spectrum (66). As a consequence trigonometric and hyperbolic quasiexactly solvable systems (see potentials 5 and 8 in Ref. 61) are generated in the form

$$
\begin{aligned}
& \frac{d^{2} X}{\mathrm{~d} \nu^{2}}+\left[\left(\frac{\alpha^{2}}{4}+\alpha\left(2 n+2 \pm k_{1} \pm k_{2}\right)\right) \cosh ^{2} \nu-\frac{\alpha^{2}}{4} \cosh ^{4} \nu-\frac{k_{1}^{2}-\frac{1}{4}}{\sinh ^{2} \nu}+\frac{k_{2}^{2}-\frac{1}{4}}{\cosh ^{2} \nu}+\lambda\right] X=0, \\
& \frac{d^{2} Y}{\mathrm{~d} \mu^{2}}-\left[\left(\frac{\alpha^{2}}{4}+\alpha\left(2 n+2 \pm k_{1} \pm k_{2}\right)\right) \cos ^{2} \mu-\frac{\alpha^{2}}{4} \cos ^{4} \mu+\frac{k_{1}^{2}-\frac{1}{4}}{\cos ^{2} \mu}+\frac{k_{2}^{2}-\frac{1}{4}}{\sin ^{2} \mu}+\lambda\right] Y=0,
\end{aligned}
$$

where $\alpha=D^{2} \omega / 2$. Thus we have established that an integral part of the notion of quasiexact solvability is the reduction of superintegrable systems to one-dimensional problems.

Indeed, we can express our observation in the form of the following hypothesis: All quantummechanical problems which are expressible as one-dimensional quasiexactly solvable systems can be determined via separation of variables in an $N$-dimensional Schrödinger equation for superintegrable systems.

This analogy prompts us to use the term quasiexact solvability for the equations of type (30) or (76), defined in the complex plane and which are not exactly solvable but which admit polynomial solutions. Thus we suggest calling quantum mechanical systems first-order quasiexactly solvable if the polynomial solution of the one-parametric differential equation of the kind of Schrödinger equation or $N$-dimensional equation after separation of variables is defined through recurrence relations which must always contain three terms or more and the discrete eigenvalues can be calculated as the solutions of algebraic equations. According to this definition systems (30) and (76) are first order quasiexactly solvable.

In three dimensions we have provided even more striking examples of 1D QES problems obtained as restrictions from superintegrable systems. We exhibited a quasiexactly solvable superintegrable system which is not at the same time exactly solvable in any separable set of coordinates. In one set of separable coordinates we obtain Ushveridze's tenth-order polynomial QES problem and in another set a fourth-order polynomial QES problem. We have shown how the eigenvalues of the symmetry operators which describe separation can be calculated from a determinant condition. These examples, and more to come on other manifolds and in higher dimensional spaces, indicate that our modified definition of QES systems can be extended to $N$-dimensional spaces and fine tuned to distinguish between the number of parameters in the systems. These matters will be taken up in other papers in this series.

\section{ACKNOWLEDGMENTS}

The work of G.S.P. was supported by the Dirección General de Asuntos del Personal Académico, Universidad Nacional Autónoma de México (DGAPA-UNAM) through Grant No. 102603 Optica Matemática and also SEP-CONACYT project 44845 and Proekto de PROMEP/ $103.5 / 05 / 1705 /$.

\section{APPENDIX: ASYMPTOTIC BEHAVIOR OF COEFFICIENTS}

To understand the behavior of the solutions of relations (36) for large $s$ we use continued fractions theory. ${ }^{81}$ Setting 


$$
\begin{aligned}
& \frac{A_{s}}{A_{s-1}}=\xi_{s} f(s), \\
& f(s)=\sqrt{\frac{\omega}{2}} \frac{\Gamma\left(\frac{s}{2}+\frac{1}{2}\right) \Gamma\left( \pm \frac{k_{2}}{2}+\frac{s}{2}+\frac{1}{2}\right) \Gamma\left(\frac{\alpha}{2 \omega} \pm \frac{k_{2}}{2}+\frac{s}{2}+1\right)}{\Gamma\left(\frac{s}{2}+1\right) \Gamma\left( \pm \frac{k_{2}}{2}+\frac{s}{2}+1\right) \Gamma\left(\frac{\alpha}{2 \omega} \pm \frac{k_{2}}{2}+\frac{s}{2}+\frac{1}{2}\right)},
\end{aligned}
$$

where $\Gamma(z)$ is the gamma function, we can write the recurrence relation (36) in the standard form

$$
\begin{aligned}
& \xi_{s}=\frac{1}{b_{s}+\xi_{s+1}}, \\
& b_{s}=\sqrt{\frac{2}{\omega}} \frac{\Gamma\left(\frac{s}{2}+\frac{1}{2}\right) \Gamma\left( \pm \frac{k_{2}}{2}+\frac{s}{2}+\frac{1}{2}\right) \Gamma\left(\frac{\alpha}{2 \omega} \pm \frac{k_{2}}{2}+\frac{s}{2}+1\right)\left[-\lambda+\frac{k_{1}}{\omega}\left(2 s+1 \pm k_{2}\right)\right]}{\Gamma\left(\frac{s}{2}+1\right) \Gamma\left( \pm \frac{k_{2}}{2}+\frac{s}{2}+1\right) \Gamma\left(\frac{\alpha}{2 \omega} \pm \frac{k_{2}}{2}+\frac{s}{2}+\frac{3}{2}\right) 2^{3}},
\end{aligned}
$$

where $\alpha=-\left(E+k_{1} /\left(8 \omega^{2}\right)\right) / 2$. Note that

$$
f(s+1) f(s)=\omega \frac{\left(\frac{\alpha}{\omega} \pm k_{2}+s+1\right)}{(s+1)\left( \pm k_{2}+s+1\right)} .
$$

Stirling's formula for the gamma function $\Gamma(z)=z^{z-1 / 2} e^{-z} \sqrt{2 \pi}(1+O(1 / z))$ as $|z| \rightarrow \infty$ with $|\arg z|$ $<\pi$, gives $f(s)=-\sqrt{\omega / s}(1+O(1 / s))$ and $b_{s}= \pm\left(k_{2} / 2 \omega \sqrt{s \omega}\right)(1+O(1 / s))$. In the following we take $k_{1} \leqslant 0, k_{2}, \lambda, E$ real and $\omega>0$. Without loss of generality we can assume $b_{s}$ is positive for sufficiently large $s$ since, otherwise, we could make the replacements $b_{s} \rightarrow-b_{s}, \xi_{s} \rightarrow-\xi_{s}$.

Since $\Sigma b_{s}=\infty$, it is a consequence of the Seidel-Stern theorem that the formal continued fraction expressions for the $\xi_{s}$ converge,

$$
\xi_{s}=\frac{1}{b_{s}}+\frac{1}{b_{s+1}}+\cdots+\frac{1}{b_{s+k}}+\cdots .
$$

Moreover, standard continued fraction theory tells us that

$$
\xi_{s}=\lim _{n \rightarrow \infty} \frac{A_{n}^{(s)}}{B_{n}^{(s)}},
$$

where

$$
\left(\begin{array}{c}
A_{-1}^{(s)} \\
B_{-1}^{(s)}
\end{array}\right)=\left(\begin{array}{l}
1 \\
0
\end{array}\right), \quad\left(\begin{array}{c}
A_{0}^{(s)} \\
B_{0}^{(s)}
\end{array}\right)=\left(\begin{array}{l}
0 \\
1
\end{array}\right),
$$

and

$$
\left(\begin{array}{c}
A_{n}^{(s)} \\
B_{n}^{(s)}
\end{array}\right)=b_{n+s}\left(\begin{array}{c}
A_{n-1}^{(s)} \\
B_{n-1}^{(s)}
\end{array}\right)+\left(\begin{array}{c}
A_{n-2}^{(s)} \\
B_{n-2}^{(s)}
\end{array}\right), \quad n \geqslant 1 .
$$

Furthermore the relation $A_{n}^{(s)} B_{n-1}^{(s)}-A_{n-1}^{(s)} B_{n}^{(s)}=(-1)^{n-1}$ holds for all $n \geqslant 0$, which implies 


$$
\frac{A_{n}^{(s)}}{B_{n}^{(s)}}-\frac{A_{n-1}^{(s)}}{B_{n-1}^{(s)}}=\frac{(-1)^{n-1}}{B_{n-1}^{(s)} B_{n}^{(s)}}
$$

This result in turn implies that the sequence $A_{2 n}^{(s)} / B_{2 n}^{(s)}$ is, for large $s$ and $n$, monotone increasing in $n$ and goes to $\xi_{s}$ in the limit, whereas $A_{2 n+1}^{(s)} / B_{2 n+1}^{(s)}$ is monotone decreasing in $n$ and goes to $\xi_{s}$ in the limit. For example,

$$
\frac{A_{2 n+2}^{(s)}}{B_{2 n+2}^{(s)}}-\frac{A_{2 n}^{(s)}}{B_{2 n}^{(s)}}=\frac{b_{2 n+2+s}}{B_{2 n}^{(s)} B_{2 n+2}^{(s)}}
$$

It follows from (A3), (A5) that

$$
\xi_{s}=\frac{A_{0}^{(s)}}{B_{0}^{(s)}}+\sum_{n=1}^{\infty} \frac{b_{2 n+2+s}}{B_{2 n}^{(s)} B_{2 n+2}^{(s)}} .
$$

Simple estimates using the recurrence relations (A4) give

$$
B_{2 n}^{(s)}>1+b_{s+1} \sum_{m=1}^{n} b_{2 m+s}, \quad B_{2 n+1}^{(s)}>\sum_{m=0}^{n} b_{2 m+1+s} .
$$

Substituting these results into the identities

$$
B_{2 n}^{(s)}=\sum_{m=1}^{n} b_{2 m+s} B_{2 m-1}^{(s)}, \quad B_{2 n+1}^{(s)}=\sum_{m=0}^{n} b_{2 m+s+1} B_{2 m}^{(s)}
$$

we get refined upper bounds for $B_{2 n}^{(s)}, B_{2 n+2}^{(s)}$. We can approximate the sum $\sum_{m=s}^{n} 1 / \sqrt{m}$ by the integral $\int_{s}^{n}(1 / \sqrt{x}) \mathrm{d} x$ and use similar approximations to get an upper bound for the series (A6),

$$
\left|\xi_{s}\right|<\kappa_{1} \int_{0}^{\infty} \frac{\mathrm{d} y}{\sqrt{y+s}\left(y^{2}+1\right)}+\kappa_{2}
$$

for positive constants $\kappa_{j}$ independent of $s$. This shows that $\left|\xi_{s}\right|$ is uniformly bounded in $s$. Since $\xi_{s+1}=-b_{s}+1 / \xi_{s}$ and $b_{s} \rightarrow 0$ as $s \rightarrow \infty$ it is also true that $\left|1 / \xi_{s}\right|$ is uniformly bounded in $s$.

It follows from (A2) that

$$
\xi_{s+1}-\xi_{s-1}=\frac{\xi_{s-1}-\left(b_{s}+\xi_{s-1}\right)\left(1-b_{s-1} \xi_{s-1}\right)}{1-b_{s-1} \xi_{s-1}} .
$$

Now choose $s_{0}$ so large that $b_{s+1}<b_{s}$ and $b_{s} \xi_{s}<1$ for all $s \geqslant s_{0}$. Note from this identity that if $\xi_{s_{1}-1}>1$ for some $s_{1}>s_{0}$ then $\xi_{s_{1}+1}>\xi_{s_{1}-1}>1$. Thus the sequence $\xi_{s_{1}+2 k-1}$ is monotonically increasing for all $k \geqslant 0$. Since $\left|\xi_{s}\right|$ is bounded, it follows that in this case $\lim _{k \rightarrow \infty} \xi_{s_{1}+2 k-1}=\xi_{+}$exists, and $\xi_{+}>1$. Since $\xi_{s+1}=-b_{s}+1 / \xi_{s}, b_{s} \rightarrow 0$ as $s \rightarrow \infty$ and $\left|1 / \xi_{s}\right|$ is uniformly bounded in $s$, then the sequence $\xi_{s_{1}+2 k}$ is also convergent, $\lim _{k \rightarrow \infty} \xi_{s_{1}+2 k}=\xi_{-}$where $0<\xi_{-}<1$.

The other possibility is that $\xi \leqslant 1$ for all $s \geqslant s_{0}$. Since $1 / \xi_{s}-\xi_{s+1}=b_{s} \rightarrow 0$ as $s \rightarrow \infty$, and $1 / \xi_{s}$ $\geqslant 1, \xi_{s+1} \leqslant 1$ for all $s \geqslant s_{0}$ it follows that $\lim _{k \rightarrow \infty} \xi_{k}=\xi_{+}=\xi_{-}=1$. Thus in all cases the sequences $\xi_{2 k}$ and $\xi_{2 k+1}$ converge.

We conclude that

$$
\frac{A_{s+1}}{A_{s}}=f(s+1) \xi_{s}=\sqrt{\frac{\omega \xi_{ \pm}}{s}}(1+O(1 / s)), \quad \xi_{+} \xi_{-}=1,
$$

depending on whether $s$ is even or odd.

${ }^{1}$ S. R. Wojciechowski, Phys. Lett. A 95, 279 (1983).

${ }^{2}$ L. P. Eisenhart, Phys. Rev. 74, 87 (1948). 
${ }^{3}$ W. Miller, Jr., Symmetry and Separation of Variables (Addison-Wesley, Providence, RI, 1977).

${ }^{4}$ E. G. Kalnins, Separation of Variables for Riemannian Spaces of Constant Curvature, Pitman, Monographs and Surveys in Pure and Applied Mathematics 28 (Longman, Essex, England, 1986).

${ }^{5}$ E. G. Kalnins, J. M. Kress, and W. Miller, J. Math. Phys. 46, 053509 (2005)

${ }^{6}$ E. G. Kalnins, J. M. Kress, and W. Miller, J. Math. Phys. 46, 053510 (2005).

${ }^{7}$ E. G. Kalnins, J. M. Kress, and W. Miller, J. Math. Phys. 46, 103507 (2005).

${ }^{8}$ E. G. Kalnins, J. M. Kress, and W. Miller (unpublished).

${ }^{9}$ J. Friš, V. Mandrosov, Ya. A. Smorodinsky, M. Uhlir, and P. Winternitz, Phys. Lett. 16, 354 (1965).

${ }^{10}$ J. Friš, Ya. A. Smorodinskii, M. Uhlír, and P. Winternitz, Sov. J. Nucl. Phys. 4, 444 (1967).

${ }^{11}$ A. A. Makarov, Ya. A. Smorodinsky, Kh. Valiev, and P. Winternitz, Nuovo Cimento A 52, 1061 (1967).

${ }^{12}$ N. W. Evans, Phys. Rev. A 41, 5666 (1990); J. Math. Phys. 32, 3369 (1991); Phys. Lett. A 147, 483 (1990).

${ }^{13}$ C. Grosche, G. S. Pogosyan, and A. N. Sissakian, Fortschr. Phys. 43, 523 (1995); Phys. Part. Nucl. 27, 244 (1996); 28, 486 (1997).

${ }^{14}$ E. G. Kalnins, J. M. Kress, W. Miller, Jr., and G. S. Pogosyan, J. Phys. A 34, 4705 (2001).

${ }^{15}$ E. G. Kalnins, W. Miller, Jr., and G. S. Pogosyan, Phys. At. Nucl. 65, 1033 (2002).

${ }^{16}$ E. G. Kalnins, W. Miller, Jr., and G. S. Pogosyan, J. Phys. A 33, 6791 (2000).

${ }^{17}$ E. G. Kalnins, W. Miller, Jr., and G. S. Pogosyan J. Phys. A 33, 4105 (2000).

${ }^{18}$ C. Grosche, G. S. Pogosyan, and A. N. Sissakian, Fortschr. Phys. 43, 453 (1995).

${ }^{19}$ E. G. Kalnins, W. Miller, Jr., and G. S. Pogosyan, J. Math. Phys. 37, 6439 (1996).

${ }^{20}$ Ye. M. Hakobyan, M. Kibler, G. S. Pogosyan, and A. N. Sissakian, Phys. At. Nucl. 61, 1782 (1998) [in Russian, Yad. Fiz. 61, 1893 (1998)].

${ }^{21}$ E. G. Kalnins, G. Williams, W. Miller, Jr., and G. S. Pogosyan, J. Math. Phys. 40, 708 (1999)

${ }^{22}$ E. G. Kalnins, W. Miller, Jr., and G. S. Pogosyan, J. Math. Phys. 38, 5416 (1997); Ye. M. Hakobyan, E. G. Kalnins, W. Miller, Jr., and G. S. Pogosyan, ibid. 40, 2291 (1999).

${ }^{23}$ M. F. Rañanda and M. Santander, J. Math. Phys. 40, 5026 (1999).

${ }^{24}$ E. G. Kalnins, W. Miller, Jr., G. S. Pogosyan, and G. C. Williams, J. Phys. A 35, 4755 (2002).

${ }^{25}$ J. M. Kress and E. G. Kalnins, Phys. At. Nucl. 65, 1080 (2002).

${ }^{26}$ E. G. Kalnins, J. M. Kress, and P. Winternitz, J. Math. Phys. 43, 970 (2002).

${ }^{27}$ A. Ballesteros, F. Herranz, M. Santander, and T. Sanz-Gil, J. Phys. A 36, L93 (2003).

${ }^{28}$ C. Gonera, J. Phys. A 37, 4085 (2004).

${ }^{29}$ E. G. Kalnins, J. M. Kress, W. Miller, and P. Winternitz, J. Math. Phys. 44, 5811 (2003).

${ }^{30}$ S. Gravel, J. Math. Phys. 45, 1003 (2004).

${ }^{31}$ A. O. Barut, A. Inomata, and G. Junker, J. Phys. A 20, 6271 (1987).

${ }^{32}$ A. O. Barut, A. Inomata, and G. Junker, J. Phys. A 23, 1179 (1990).

${ }^{33}$ E. T. Whittaker and G. N. Watson A Course of Modern Analysis (Cambridge University Press, Cambridge, 1927), Vol. II.

${ }^{34}$ P. W. Higgs, J. Phys. A 12, 309 (1979).

${ }^{35}$ H. I. Leemon, J. Phys. A 12, 489 (1979).

${ }^{36}$ Ya. A. Granovsky, A. S. Zhedanov, and I. M. Lutzenko, J. Phys. A 24, 3887 (1991).

${ }^{37}$ P. Letourneau and L. Vinet, Ann. Phys. 243, 144 (1995).

${ }^{38}$ D. Bonatos, C. Daskaloyannis, and K. Kokkotas, Phys. Rev. A 50, 3700 (1994).

${ }^{39}$ C. Daskaloyannis, J. Math. Phys. 40, 1100 (2001).

${ }^{40}$ Superintegrability in Classical and Quantum Systems, edited by P. Tempesta, P. Winternitz, W. Miller, and G. Pogosyan (AMS, Providence, RI, 2005), Vol. 37.

${ }^{41}$ P. Tempesta, A. Turbiner, and P. Winternitz, J. Math. Phys. 42, 4248 (2001).

${ }^{42}$ M. Rodriguez and P. Winternitz, J. Math. Phys. 43, 1309 (2002).

${ }^{43}$ D. Gomez-Ullate, N. Kamran, and R. Milson, J. Phys. A 38, 2005 (2005).

${ }^{44}$ S. Flügge, Problems in Quantum Mechanics (Springer-Verlag, Berlin, Heidelberg, New York, 1971), Vol. 1.

${ }^{45}$ O. L. de Lange and R. E. Raab, Operator Methods in Quantum Mechanics (Claredon, Oxford, 1991).

${ }^{46}$ G. A. Natanzon, Theor. Math. Phys. 38, 146 (1979).

${ }^{47}$ Higher Transcendental Functions, edited by A. Erdélyi, W. Magnus, F. Oberhettinger, and F. G. Tricomi (McGraw-Hill, New York, 1955), Vols. I-III.

${ }^{48}$ N. M. Atakishiev, G. S. Pogosyan, L. E. Vicent, and K. B. Wolf, J. Phys. A 34, 9381 (2001); 34, 9399 (2001).

${ }^{49}$ B. D. Sleeman, Multiparameter Spectral Theory in Hilbert Space (Research Notes in Mathematics No. 22) (Pitman, London, 1978).

${ }^{50}$ E. L. Ince, Ordinary Differential Equation (Dover, New York, 1956).

${ }^{51}$ A. H. Wilson, Proc. R. Soc. London, Ser. A 118, 617 (1928).

${ }^{52}$ G. S. Pogosyan and P. Winternitz, J. Math. Phys. 43, 3387 (2002).

${ }^{53}$ L. G. Mardoyan, G. S. Pogosyan, A. N. Sissakian, and V. M. Ter-Antonyan, J. Phys. A 18, 455 (1985).

${ }^{54}$ V. A. Dulock and H. V. McIntosh, Am. J. Phys. 33, 109 (1969).

${ }^{55}$ M. J. Englefield, Group Theory and the Coulomb Problem (Wiley-Interscience, New York, London, Sydney, Toronto, 1972).

${ }^{56}$ L. G. Mardoyan, G. S. Pogosyan, A. N. Sissakian, and V. M. Ter-Antonyan, Theor. Math. Phys. 61, 1021 (1984).

${ }^{57}$ I. V. Komarov, L. I. Ponomarev, and S. Y. Slavyanov, Spheroidal and Coulomb Spheroidal Functions (Nauka, Moscow, 1996).

${ }_{59}^{58}$ A. A. Bogush and V. S. Otchik, J. Phys. A 30, 559 (1997).

${ }^{59}$ A. V. Turbiner and A. G. Ushveridze (unpublished). 
${ }^{60}$ A. V. Turbiner, Commun. Math. Phys. 118, 467 (1988).

${ }^{61}$ A. G. Ushveridze, Sov. J. Part. Nucl. 20, 504 (1989).

${ }^{62}$ M. A. Shifman, Int. J. Mod. Phys. A 126, 2897 (1989).

${ }^{63}$ A. G. Ushveridze, Quasi-Exactly Solvable Models in Quantum Mechanics (Institute of Physics, Bristol, 1993).

${ }^{64}$ V. Singh, S. N. Biswas, and K. Dutta, Phys. Rev. D 18, 1901 (1978).

${ }^{65}$ A. V. Turbiner and A. G. Ushveridze, Phys. Lett. A 126, 181 (1987).

${ }^{66}$ C. M. Bender and G. V. Dunne, hep-th/9511138.

${ }^{67}$ Ye. M. Hakobyan, G. S. Pogosyan, A. N. Sissakian, and S. I. Vinitsky, Phys. At. Nucl. 62, 623 (1999) [in Russian, Yad. Fiz. 62, 671 (1999)].

${ }^{68}$ R. G. Airapetyan, Kh. G. Karayan, G. S. Pogosyan, A. N. Sissakian, and D. I. Zaslavsky (unpublished).

${ }^{69}$ L. G. Mardoyan, G. S. Pogosyan, A. N. Sissakian, and V. M. Ter-Antonyan, Nuovo Cimento B 88, 43 (1985).

${ }^{70}$ L. S. Davtyan, L. G. Mardoyan, G. S. Pogosyan, A. N. Sissakian, and V. M. Ter-Antonyan (unpublished).

${ }^{71}$ P. Kustaanheimo and E. Stiefel, J. Reine Angew. Math. 218, 204 (1965).

${ }^{72}$ I. I. Goldman and D. V. Krivchenkov, Problems in Quantum Mechanics (Pergamon, London, 1961).

${ }^{73}$ L. D. Landau and E. M. Lifshitz, Quantum Mechanics: Non-relativistic Theory (Pergamon, London, 1981).

${ }^{74}$ R. L. Hall, N. Saad, and A. B. von Keviczky, J. Math. Phys. 43, 94 (2002).

${ }^{75}$ F. Calogero, J. Math. Phys. 10, 2191 (1969).

${ }^{76}$ S. B. Isakov, Int. J. Mod. Phys. A 9, 2563 (1994).

${ }^{77}$ Ye. Hakobyan and V. M. Ter-Antonyan, quant-ph/00002069.

${ }^{78}$ C. A. Coulson and P. D. Robertson, Proc. Phys. Soc. London 71, 815 (1958).

${ }^{79}$ R. Courant and D. Hilbert, Methods of Mathematical Physics (Intersciences, New York, 1953), Vol. I, Chap. 6.

${ }^{80}$ F. M. Arscott, Proc. R. Soc. Edinburgh, Sect. A: Math. Phys. Sci. 67, 265 (1967).

${ }^{81}$ L. Lorentzen and H. Waadeland, Continued Fractions with Applications (North-Holland, Amsterdam, 1992). 